\title{
HyperChIP for identifying hypervariable signals across ChIPIATAC-seq samples
}

Haojie Chen ${ }^{1,2 \#}$, Shiqi Tu ${ }^{1 \# *}$, Chongze Yuan ${ }^{3}$, Feng Tian ${ }^{1,2}$, Yijing Zhang $^{4}$, Yihua Sun ${ }^{3}$, Zhen Shao ${ }^{1,2 *}$

${ }^{1}$ CAS Key Laboratory of Computational Biology, Shanghai Institute of Nutrition and Health, Chinese Academy of Sciences, Shanghai 200031, China.

2University of Chinese Academy of Sciences, Beijing 100049, China.

${ }^{3}$ Department of Thoracic Surgery and State Key Laboratory of Genetic Engineering, Fudan University Shanghai Cancer Center, Shanghai 200032, China.

${ }^{4}$ State Key Laboratory of Genetic Engineering, Collaborative Innovation Center of Genetics and Development, Department of Biochemistry, Institute of Plant Biology, School of Life Sciences, Fudan University, Shanghai 200438, China.

\#These authors contributed equally to this work.

\section{*Corresponding to:}

Dr. Shiqi Tu

Email: tushiqi@picb.ac.cn

\section{Dr. Zhen Shao}

Email: shaozhen@picb.ac.cn

\author{
Abstract \\ With the reduction in sequencing costs, studies become prevalent that profile the \\ chromatin landscape for tens or even hundreds of human individuals by using \\ ChIP/ATAC-seq techniques. Identifying genomic regions with hypervariable \\ ChIP/ATAC-seq signals across given samples is essential for such studies. In \\ particular, the hypervariable regions (HVRs) across tumors from different patients \\ indicate their heterogeneity and can contribute to revealing potential cancer subtypes \\ and the associated epigenetic markers. We present HyperChIP as the first complete \\ statistical tool for the task. HyperChIP uses scaled variances that account for the \\ mean-variance dependence to rank genomic regions, and it increases the statistical \\ power by diminishing the influence of true HVRs on model fitting. Applying it to a \\ large pan-cancer ATAC-seq data set, we found that the identified HVRs not only \\ provided a solid basis to uncover the underlying similarity structure among the \\ involved tumor samples, but also led to the identification of transcription factors \\ pertaining to the similarity structure when coupled with a motif-scanning analysis.
}




\section{Introduction}

Chromatin immunoprecipitation followed by high-throughput sequencing (ChIP-seq) is the premier technology for profiling genome-wide localization of chromatin-binding proteins, including transcription factors and histones with various modifications [1, 2]. Besides, assay for transposase-accessible chromatin using sequencing (ATAC-seq) has been widely adopted for detection of open chromatin [3]. As a common computational task for learning from ChIP/ATAC-seq data, identifying genomic regions with significant changes of ChIP/ATAC-seq signal intensities across samples is essential to understanding the epigenetic mechanisms that orchestrate the variation of gene expression program [4-6]. For this task, there are two major analyses suited to distinct application scenarios. The first one is referred to as differential analysis, in which the label of each sample is clearly defined (e.g., healthy or diseased) and differential ChIPIATAC-seq signals between different labels are identified by comparing the corresponding samples [7, 8]. The second analysis does not require prior knowledge regarding the labels of samples and aims at identifying hypervariable ChIPIATAC-seq signals across samples, which can then be used as features for a classification of the samples. This analysis is of particular importance in classifying samples of different cancer patients and gaining insights into epigenetic markers of different cancer subtypes/stages $[6,9,10]$. Since the second analysis is intrinsically an unsupervised one, it typically requires many more samples to achieve reliable results compared to the first analysis.

In early years, the practical applicability of hypervariable analysis was seriously limited by the number of ChIP-seq samples available in a study, and researchers were more inclined to apply differential analysis with a proper experimental design. As a result, a large body of mature computational tools has been developed for differential ChIP-seq analysis [7, 8]. A contrasting example is the analysis of singlecell RNA-seq (scRNA-seq) data. As an individual scRNA-seq experiment generates transcriptome profiles of a large number of cells and researchers typically have no detailed prior knowledge about the cell identities, hypervariable analysis has been frequently applied to scRNA-seq data [11-13]. Accordingly, many computational tools for identifying hypervariable genes (HVGs) from scRNA-seq data and using these genes to classify cells have been developed [14-16].

In recent years, with the decrease of sequencing costs, there are more and more large-scale studies in which tens or even hundreds of ChIP/ATAC-seq profiles for different human individuals are generated, and hypervariable analysis is becoming increasingly prevalent in the analysis of ChIP/ATAC-seq data $[6,9,10,17,18]$. In particular, hypervariable ChIP/ATAC-seq signals across cancer patients could be potential epigenetic markers of different cancer subtypes/stages, and these markers may serve as therapeutic targets and may contribute to the prognosis of patients [6]. 
To our best knowledge, however, there are currently no such computational tools that are specifically developed for hypervariable ChIPIATAC-seq analysis.

In many studies, researchers designed their own computational pipelines for calling hypervariable ChIP/ATAC-seq signals [9, 10, 17, 19-22], but some of these pipelines failed to take some basic data characteristics into account. For example, sequencing count data are inherently associated with a strong dependence of signal variability on the mean signal intensity, making the ChIP/ATAC-seq signal variability of different genomic regions not directly comparable with each other. Specifically, after a logarithmic transformation, small log-counts tend to have larger variances than large log-counts $[23,24]$. While this mean-variability relationship has been properly accounted for in almost all the tools for calling HVGs from scRNA-seq data, several studies called hypervariable ChIP/ATAC-seq signals by ranking genomic regions based on some variability index without considering its dependence on the mean signal intensity $[9,10,19,21,22]$. Other studies alleviated the influence of the mean-variability trend by applying various practical strategies, such as combining the rankings based on mean intensities and variances [20], and making a logtransformation with a large offset count to suppress the large variances of small logcounts [17]. These strategies were effective, but their implementation details (e.g., the exact offset count) and performance highly rely on the specific data set and, thus, their general applicability is questionable.

A more fundamental problem is that, instead of coming up with a probabilistic model to assess the statistical significances of observed ChIP/ATAC-seq signal variability, all these studies ranked genomic regions and selected a certain number or proportion of top-ranked ones as hypervariable regions (HVRs), with the specific number or proportion being determined based on practical experience. In these studies, the numbers and proportions ranged from 1,000 to 10,000 and from $1 \%$ to $25 \%$, respectively. On the one hand, designing a complete statistical model with $p$ value calculation can increase the adaptivity to various data sets and avoid an arbitrary selection of HVRs. On the other hand, using an ordinary model fitting framework will almost certainly lead to conserved $p$-values and low statistical power for identifying HVRs, since it is difficult to strictly avoid the influence of true HVRs on the fitting process. A previous study of ours has suggested that $p$-values derived from an ordinary parameter estimation framework can hardly bear the strength of multiple testing adjustment [6].

In this study, we present HyperChIP, a statistical method for hypervariable ChIPIATAC-seq analysis that is aimed at addressing the above concerns. In this method, a variability statistic that has been corrected for the mean signal intensity is used as the key statistic, and specific efforts have been made to increase the statistical power for identifying HVRs. For the latter, HyperChIP selects a subset of 
genomic regions with relatively low signal intensities for parameter estimation, and further employs the Winsorization technique [25] to render the estimation procedure robust to true HVRs. Our empirical observations on various data sets suggest that these low-intensity regions contain only a small proportion of HVRs, which can be effectively handled by Winsorization. Applying HyperChIP to several real data sets, of which each comprised ChIPIATAC-seq profiles of tens of cancer patients, we found that the method can identify hundreds to thousands of significant HVRs at common cutoffs of the $\mathrm{BH}$-adjusted $p$-value, which controls the false discovery rate [26]. Further exploration revealed that the identified HVRs tended to be associated with the tumor progression stages of patients. We also observed a systematic difference in variability structure between proximal and distal regions. Specifically, the ChIP/ATAC-seq signal variability in distal regions was considerably higher than that in proximal regions, which was consistent with previous studies showing that the activity of enhancer elements is much more variable across individuals and cellular contexts than is the activity of gene promoters [27-29]. We therefore highlight the necessity of separately dealing with proximal and distal regions in a hypervariable ChIP/ATAC-seq analysis, to avoid the suppression of the statistical power for identifying proximal HVRs.

To demonstrate the practical utility of HyperChIP, we additionally applied it to a pan-cancer ATAC-seq data set, which was generated by The Cancer Genome Atlas (TCGA) program and consisted of ATAC-seq profiles of tumors from hundreds of patients across 23 cancer types [17]. Based on the identified HVRs, we investigated the similarity structure among the ATAC-seq profiles. While most of them were well clustered by their cancer types, those of various types of squamous cell carcinoma (SC) tended to be mixed up with each other. We further defined super classes of cancer types based on the similarity structure and identified transcription factors (TFs) specific to each class by applying a motif-scanning procedure on the HVRs. Notably, many of the identified TFs were found to be exclusively expressed in the corresponding classes showing a strong association with either tissue specificity or tumorigenesis or both. For example, TP63, a confirmed oncogene in several SC types [30, 31], was identified as the most significant TF specific to the SC class.

\section{Results}

\section{Ranking genomic regions based on scaled variances}

Three data sets were used to evaluate the performance of HyperChIP (Table 1). The first data set comprised H3K27ac ChIP-seq profiles, a histone modification marking active gene promoters and distal enhancers, of tumor tissues of 36 lung adenocarcinoma (LUAD) patients [6]. The second one consisted of ATAC-seq 
samples of 34 non-small cell lung carcinoma (NSCLC) patients [18]. The third one consisted of RNA polymerase (Pol) II ChIP-seq samples of 26 LUAD cell lines derived from different patients [32].

To facilitate the understanding of how HyperChIP works, we briefly describe the required input data of it. HyperChIP takes a matrix of normalized $\log _{2}$ read counts as input. Rows and columns of the matrix correspond to a pre-defined list of genomic regions and a set of ChIP-seq samples, respectively. In this study, we separately compiled a list of regions for each data set. Given a data set, we first called peaks for each sample and merged all the resulting peaks. Broad merged peaks were then divided up into consecutive bins, and narrow ones were left as they were. As for normalization, we constructed a pseudo-reference profile by averaging all the samples, and invoked the MA normalization procedure implemented in MAnorm2 [24] to normalize each sample against it (see Methods). Note also that, unless otherwise stated, each hypervariable analysis in this study targeting an individual data set has separately handled proximal and distal regions.

Given a matrix of normalized signal intensities, HyperChIP accounts for the associated mean-variability relationship by applying a gamma-family regression method to observed mean-variance pairs. Specifically, it employs a local regression procedure to allow for general mean-variance relationships [33]. It can be easily seen that all the three data sets are associated with clear mean-variance dependence, and the specific trend depicted by the fitted mean-variance curve (MVC) varies across the data sets (Fig. 1A and Supplementary Fig. 1). Then, HyperChIP uses the MVC to derive a scaled variance for each genomic region, which is defined as the ratio of the observed variance to the predicted variance obtained from the MVC (Fig. 1B). These scaled variances shall be used for ranking regions and selecting HVRs.

To benchmark HyperChIP, we considered several other methods for ranking regions. These methods can be classified into two classes. One class uses some variability statistic to rank regions without taking its dependence on the mean signal intensity into account. Such statistics included the observed (unscaled) variance [19, 21, 22], median absolute deviation (MAD) [9], and interquartile range (IQR) [10]. We found that all the methods of this class were associated with a tendency to select regions with low signal intensities as HVRs (Supplementary Fig. 2A). Methods of the other class adopt different practical strategies to account for the mean-variability dependence. We included two methods in this class. The first method, referred to as min-rank, is primarily aimed at selecting regions with both high intensities and high variability [20]. It first separately uses the observed mean intensities and the observed variances to sort genomic regions into ascending order. Then, it takes the minimum of the two ranks associated with each region, and uses these minima to once again rank the regions. When applying min-rank, we found this method clearly 
tended to select regions with high intensities as HVRs (Supplementary Fig. 2B). The second method follows a computational pipeline used in a previous study [17]. It first derives CPM (count per million) values and applies a $\log _{2}$ transformation with a moderately large offset. The results are then subject to quantile normalization. For this method, we have separately tried 5 and 10 as the offset value (Supplementary Fig. 2C).

To assess the rankings of genomic regions provided by each method, we calculated true discovery proportions (TDPs) among top-ranked regions. More specifically, we defined, among proximal regions, true HVRs as those regions that were linked with HVGs, which were identified based on the corresponding RNA-seq data (see Methods and Supplementary Fig. 3). We then plotted the TDP against the number of top-ranked proximal regions for each method. Compared with the methods that do not consider the mean-variability dependence, HyperChIP achieved much higher TDPs for all the three data sets (Fig. 1C). Compared with the methods of the other class, HyperChIP performed better or as well as them depending on the specific data set (Fig. 1D).

A common downstream analysis based on identified HVRs is to use them as features for a classification of samples, with the hope of revealing the substructure of the samples. In the ATAC-seq data set, the 34 NSCLC patients consisted of 26 LUAD and 8 LUSC (lung squamous cell carcinoma) cases, corresponding to two primary subtypes of NSCLC [18]. These subtype labels can be considered gold-standard for evaluating classifications of the patients. Here, based on top-ranked HVRs derived by different methods, we performed classifications of the patients into two sub-groups (see Methods) and assessed the consistency between the classifications and the gold standard by using the adjusted Rand index (ARI) [34]. This index has an expected value of 0 for random classifications, and is bounded above by 1 for a perfect agreement between two classifications. The relative performance of different methods was similar as in the previous comparison based on HVGs: HyperChIP showed an overall performance better than each of the other methods, especially when it was compared with the methods that do not consider the mean-variability dependence (Fig. 2A, B). Furthermore, the classification results from HyperChIP were very stable across a wide range of numbers of used HVRs (Fig. 2C).

\section{Modeling scaled variances and increasing the statistical power for identifying HVRs}

A complete statistical model specifying the null distribution of the scaled variances is required for assessing their statistical significances. We previously developed MAnorm2 for differential ChIP-seq analysis [24]. In HyperChIP, we follow the distributional theory proposed by MAnorm2 for modeling a group of ChIP-seq 
samples. Specifically, we assume the normalized $\log _{2}$ read counts at each genomic region follow a normal distribution, with the precision parameter associated with a prior gamma distribution whose expectation value is determined by the MVC.

Formally, let $X_{i j}$ denote the normalized $\log _{2}$ read count at region $i$ in sample $j$. We assume

$$
\begin{aligned}
X_{i j} \mid \sigma_{i}^{2} & \sim N\left(\mu_{i}, \gamma \sigma_{i}^{2}\right), \\
\frac{1}{\sigma_{i}^{2}} & \sim \frac{1}{f\left(\mu_{i}\right)} \cdot \frac{\chi_{d_{0}}^{2}}{d_{0}} .
\end{aligned}
$$

Here, $\mu_{i}$ and $\sigma_{i}^{2}$ are two unknown parameters that quantify the mean signal intensity at region $i$ and the associated signal variability, respectively; $f(\cdot)$ denotes the MVC; $d_{0}$, referred to as the number of prior degrees of freedom, effectively assesses how dispersedly the observed variances are distributed around the MVC (larger values of $d_{0}$ indicate the observed variances are more concentrated at the $\mathrm{MVC}) ; \gamma$ is referred to as the variance ratio factor and is designed for scaling the MVC to better fit the observed mean-variance pairs under this model formulation.

Next, suppose $\hat{\mu}_{i}$ and $\hat{t}_{i}$ are the observed mean intensity and variance of region $i$, respectively (i.e., the sample mean and sample variance of all $X_{i j}$ associated with region $i$ ). Based on the model, it follows that

$$
\frac{\hat{t}_{i}}{f\left(\mu_{i}\right)} \sim \gamma F_{m-1, d_{0}}
$$

where $m$ is the total number of samples. The scaled variances for ranking genomic regions are exactly obtained by replacing $\mu_{i}$ on the left-hand side of (3) with $\hat{\mu}_{i}$, and HyperChIP uses the right-hand side of (3) as the null distribution of the scaled variances for deriving $p$-values (i.e., upper-tailed probabilities).

Previously, we adopted in MAnorm2 a method of moments for parameter estimation, in which $d_{0}$ and $\gamma$ are estimated by matching the first two sample moments of log scaled variances with the corresponding theoretical moments of $\log \left(\gamma F_{m-1, d_{0}}\right)$. In a hypervariable analysis, however, using this method would lead to low statistical power for identifying HVRs, since true HVRs are included in the calculation of sample moments as well and their large variances would give rise to an underestimated $d_{0}$. In fact, when applying this method to the three data sets, we can barely identify any significant HVRs at common cutoffs of the $\mathrm{BH}$-adjusted $p$-value (Tables 2, 3).

This low-power problem can be alleviated by selecting a subset of genomic regions with relatively low abundance of HVRs to estimate the parameters, provided that the selection criterion is statistically independent of the test statistic (i.e., the scaled variance) under the null model. Inspired by the independent filtering strategy designed by DESeq2 for reducing the strength of multiple testing adjustment [35], HyperChIP uses the observed mean intensity of each region as its selection criterion. 
To determine the specific criterion, we first examined how top-ranked HVRs were distributed along the range of mean intensities for each of the three data sets. More specifically, we sorted all regions in ascending order (with respect to the mean intensities) and divided them into 10 equally-sized groups. We then calculated the proportion of those regions among each group that were ranked in the top 2,000 HVRs. It was found that, among proximal regions, top-ranked HVRs tended to be enriched within the groups with moderately large mean intensities, while for distal regions they tended to be enriched within the groups with the largest mean intensities (Fig. 3A and Supplementary Fig. 4A). We also noticed that previous studies had observed many genes with very stable expression strength across cellular contexts. These genes, referred to as lowly variable genes (LVGs), tended to be related to fundamental or so-called constitutive cellular processes, such as translation and translational elongation [15]. Since the regions in which ChIP-seq signals have extremely low variability across samples could also lead to the underestimation of $d_{0}$, we examined as well the distribution of top-ranked lowly variable regions (LVRs), namely the regions having the smallest scaled variances (Fig. 3B). For both proximal and distal regions, we found that the top-ranked LVRs tended to be enriched within the groups with the largest mean intensities (Fig. 3C and Supplementary Fig. 4B).

Based on these observations, we set the default behavior of HyperChIP to using the $10 \%$ of regions having the smallest mean intensities for parameter estimation. To further account for the presumably small amount of HVRs and LVRs among these low-intensity regions, HyperChIP integrated the Winsorization procedure [25] into the original moment matching method to avoid their influence on parameter estimation (see Methods). Using the three data sets as examples, we tried different parameter estimation strategies and compared the resulting $d_{0}$ estimates. With the subset selection and the use of Winsorization, a stepwise increase in the estimated $d_{0}$ was consistently observed across the data sets (Fig. 3D and Supplementary Fig. 4C), suggesting an improved statistical power associated with the model fitted by HyperChIP.

To more directly demonstrate the effect of the modifications made by HyperChIP to the original MAnorm2 method, which does not apply a subset selection and Winsorization, we compared the empirical distribution of scaled variances with the null distributions inferred by the two methods. We first paid specific attention to the low-intensity regions selected by HyperChIP for parameter estimation, and we observed that, when applying the original method, most of these regions did not even reach the variability magnitude as suggested by the null distribution (Fig. 4A), indicating this null distribution was too biased towards large values for a sensitive identification of HVRs. In comparison, the null distribution inferred by HyperChIP better matched these regions, especially for the regions with relatively small scaled 
variances (Fig. 4B). Accompanying with this improved fit to the low-intensity regions was a dramatic change of the overall distribution of $p$-values (Fig. 4C, D).

Specifically, the $p$-values resulting from the original method were generally conserved with no enrichment near 0 , while the $p$-values derived by HyperChIP showed clear enrichments at both ends of $[0,1]$, highlighting improved statistical power for identifying HVRs as well as LVRs. With this improvement, we were now able to detect hundreds to thousands of statistically significant HVRs for each of the three data sets (Tables 2, 3).

\section{Biological interpretation of HVRs and LVRs}

To explore the roles of HVRs/LVRs in specific biological contexts, we first defined a set of significant proximal HVRs/LVRs for the H3K27ac ChIP-seq data set $\mathrm{BH}$ adjusted $p$-value $<0.1$; for the identification of significant LVRs, $p$-values were derived as lower-tailed probabilities of the null distribution). Then, we performed gene ontology (GO) enrichment analysis for the genes linked with these HVRs/LVRs (only the GO terms for biological processes were used). For comparison, we also randomly selected a set of other proximal peak regions that matched the number of the HVRs. Compared to the genes linked with these randomly selected regions, from which no significant $\mathrm{GO}$ terms were enriched when relatively stringent $p$-value cutoffs were applied, the genes linked with the HVRs/LVRs enriched more terms (Fig. 5A), suggesting they might be associated with coordinated biological functions. Specifically, the genes linked with the LVRs were enriched for GO terms of constitutive cellular processes, including various histone modifications, tRNA modification, and RNA catabolic process (Fig. 5B and Supplementary Table 1). Consistently, the LVRs showed clearly higher sequence conservation across species [36] than both the HVRs and the randomly selected regions (Fig. 5C). Similar results were also observed when we defined significant distal HVRs/LVRs and selected a set of random distal peak regions (Fig. 5D).

In contrast, the genes linked with the proximal HVRs were enriched for GO terms related to lung development, cell differentiation, or the identity of lung cells (Fig. 5E and Supplementary Table 2). For example, surfactant homeostasis, which is a kind of chemical homeostasis important for the lungs, is a characteristic biological process of alveolar cells [37], a confirmed cell of origin of $\operatorname{LUAD}[38,39]$. Since alveolar cells become more and more poorly differentiated and gradually lose their cell identity as LUAD progresses [40], these results implied that the epigenetic heterogeneity at the proximal HVRs across the LUAD patients may be connected with their different tumor progression stages. Following this speculation, we examined the correlations of the H3K27ac ChIP-seq signals in different regions with the histopathological labels of the patients that can reflect their tumor progression stages (see Methods), and we 
observed considerably stronger correlations on the proximal HVRs compared to both the proximal LVRs and the random proximal peak regions (Fig. 5F). Similar results were also observed on the distal regions (Fig. 5G). We next evaluated the prognostic associations of different regions by separately performing a Cox regression on the H3K27ac ChIP-seq signal in each region [41]. It was found that the proximal/distal HVRs were more significantly associated with the survival time of the patients than the proximal/distal LVRs and the random proximal/distal peak regions (Supplementary Fig. 5A, B). Moreover, we performed a hierarchical clustering of the patients by using both the proximal and distal HVRs as features (see Methods). The patients were classified into two distinct sub-groups, and a significant survival difference was observed between them (Supplementary Fig. 5C, D).

Genetic variation across individuals has been revealed as one of the causes of the associated epigenetic heterogeneity [28, 42]. To explore this relationship, we assessed the enrichment of genetic variants within HVRs. Here, we defined significant proximal and distal HVRs for the ATAC-seq data set, and mapped them to the somatic single nucleotide variants (SNVs) identified for the associated NSCLC patients by the original study [18]. By random simulation, we observed that both the proximal and distal HVRs contained significantly more somatic SNVs than by chance (Fig. $5 \mathrm{H}, \mathrm{I}$ ). We also obtained a list of germline single nucleotide polymorphisms (SNPs) for the patients, and found a similar enrichment of them within the HVRs (Supplementary Fig. 6). Among these germline SNPs, we next performed a systematic identification of the quantitative trait loci (QTLs) at which different genotypes were associated with significantly differential ATAC-seq signals in the vicinity (see Methods). Owing to the large number of statistical tests and the relatively small sample size, only several significant QTLs were identified after multiple testing adjustment, and most of them were located within the HVRs (Supplementary Fig. 7A, $B$ ). For example, the most significant QTL was located within a proximal HVR, and its genotype was significantly associated with the ATAC-seq signal at the HVR as well as the expression strength of the downstream gene (Supplementary Fig. 7C, D). The most significant distal QTL was located within an HVR as well, and its genotype was significantly associated with both the ATAC-seq signal at the HVR and the expression strength of the nearest gene (Supplementary Fig. 7E, F). In conclusion, these findings indicated that the epigenetic heterogeneity at the HVRs across the patients was linked with their genetic variation.

\section{Applying HyperChIP to a large pan-cancer ATAC-seq data set}

To illustrate the practical utility of HyperChIP, we applied it to a pan-cancer ATAC-seq data set from TCGA [17], which comprised ATAC-seq samples of tumor tissues of 410 patients across 23 cancer types (Supplementary Table 3). Applying HyperChIP 
to this data set, we identified 5,823 proximal HVRs and 2,393 distal ones $(\mathrm{BH}$ adjusted $p$-value<0.1). Based on the ATAC-seq signals in these HVRs, we performed a t-SNE (t-distributed stochastic neighbor embedding) analysis [43] to dissect the similarity structure among the patients (see Methods). Naturally, most of the cancer types were well separated from each other in the two-dimensional t-SNE plot (Fig. 6A). We indeed, however, noticed a few exceptions. For example, KIRC (kidney renal clear cell carcinoma) and KIRP (kidney renal papillary cell carcinoma), which have the same tissue of origin [44], were very close to each other in the t-SNE plot. Another similar example was GBM (glioblastoma multiforme) and LGG (brain lower grade glioma), both of which were brain cancers. Of note, there were two mixtures of cancer types, both of which involved cancer types of different tissue origins (Fig. 6B, C). Further, we found that the two mixtures largely corresponded to SC and digestive adenocarcinoma (DIAD) respectively. Specifically, the ESCA (esophageal carcinoma) patients in this data set were comprised of 12 ESSC (esophageal squamous cell carcinoma) and 6 ESAD (esophageal adenocarcinoma) cases, and the distribution of these two subtypes in the t-SNE plot was very consistent with that of the SC and DIAD classes (Fig. 6D), suggesting the HVRs identified by HyperChIP can contribute to revealing the substructures of individual cancer types. Another example regarded the BRCA (breast invasive carcinoma) patients, which consisted of 14 basal and 61 non-basal cases. These two subtypes of BRCA corresponded to two patient clusters that were clearly separated from one another (Supplementary Fig. 8).

Next, we focused on the four super classes of cancer types (i.e., kidney carcinoma, brain cancer, SC, and DIAD) and explored the common properties of each class from the perspective of transcriptional regulators. Technically, we obtained 521 binding motifs of 432 different human TFs from the JASPAR database [45] and quantitatively inferred the activity of the TFs in each ATAC-seq sample (an individual TF could be associated with multiple motif versions). To achieve that, we first identified the instances of each motif in the genome by employing a motif-scanning routine [46]. Then, for each motif, we took the HVRs containing its instances and used the average ATAC-seq signal across these HVRs (in each sample) as an activity score of the corresponding TF (the ATAC-seq signals in different HVRs had been separately scaled beforehand; see Methods). Finally, we identified class-specific TFs by comparing the activity scores derived from each motif between the samples belonging to each class and the other samples, with the application of $t$-tests. Figure $6 \mathrm{E}$ illustrates several representative (top-ranked) motifs for each of the four classes.

We noticed that a number of the identified class-specific TFs have been reported to have tissue-specific activity in the corresponding organs. For example, HNF4A is an important TF linked with the regulation of liver-specific gene expression as well as multiple biological processes in epithelia of the gastrointestinal tract and kidneys [47, 
48]. In our analysis, both HNF4A and HNF4A.1 were identified as top-ranked motifs for the DIAD class (Fig. 6F), of which all the three cancer types originated in organs of the gastrointestinal tract (i.e., the esophagus, stomach, and large intestine). Compared to the other cancer types, ATAC-seq samples of the DIAD and kidney carcinoma classes as well as those of the liver-associated cancer types, including LIHC (liver hepatocellular carcinoma) and CHOL (cholangiocarcinoma), showed clearly higher HNF4A activity scores (Fig. 6G). Consistently, based on the matched RNA-seq samples, we found $H N F 4 A$ was expressed almost exclusively in these cancer types (Fig. 6H). We further accessed the expression strength of HNF4A in tumor tissues and adjacent normal tissues of a much larger cohort of cancer patients, by using the GEPIA web server [49]. A consistent tissue specificity of HNF4A was observed in this cohort as well, and we found no systematic differential expression of HNF4A between the tumor and matched normal tissues (Fig. 6I). Another example was HNF1A, a TF specifically expressed in organs of endoderm origin, including the kidneys and almost all the digestive organs [50]. In our analysis, HNF1A was identified as a top-ranked TF for the kidney carcinoma class, and both of its activity scores and expression strength showed consistent tissue specificity with its biological roles (Supplementary Fig. 9A-C). Again, we found no systematic differential expression of HNF1A between the tumor and matched normal tissues (Supplementary Fig. 9D).

By contrast, multiple top-ranked TFs identified for the SC class were common oncogenes shared by the involved SC types. For example, TP63 has been implicated as an oncogene in several SC types, including HNSC (head and neck squamous cell carcinoma) [51], LUSC [52], and ESSC [53]. In our analysis, TP63 was the top one TF for the SC class (Fig. 6J). Compared to the other cancer types, the cancer types of the SC class exhibited considerably higher activity scores and expression levels of TP63 (Fig. 6K, L). We next examined the expression of TP63 in the larger cohort of cancer patients. Notably, for each SC type, TP63 showed significantly higher expression in the tumor tissues than in the matched normal tissues, and such upregulation of TP63 was not observed in any non-SC cancer types (Fig. 6M). These results suggested TP63 could be a "pan-SC" oncogenic TF. Another example was TP73, which ranked second for the SC class. Similar to TP63, TP73 showed systematically increased expression in the tumor tissues of the SC types (Supplementary Fig. 10).

There was also a third type of class-specific TFs, which was associated with strong tissue specificity as well as significantly differential expression between tumor and matched normal tissues. For example, RFX4 is a TF specifically expressed in the brain [54], and it has been recently revealed to be associated with tumor progression in patients with glioblastoma [55]. In our analysis, RFX4 ranked first for the brain 
cancer class (Supplementary Fig. 11A). Among all the cancer types, RFX4 was exclusively expressed in GBM and LGG, and its expression in them was significantly higher than in the matched normal tissues (Supplementary Fig. 11B-D). Together, these results indicated the HVRs identified by HyperChIP can contribute to the identification of regulators pertaining to the heterogeneity across samples.

\section{Discussion}

With the decrease of sequencing costs, there are more and more large-scale cancer studies in which ChIPIATAC-seq profiles for tens or even hundreds of different patients are generated. Hypervariable analysis can play an essential role in such studies, considering its importance in dissecting the similarity structure among patients and discovering potential cancer subtypes. In the study, we presented HyperChIP as the first complete statistical tool that is specifically developed for identifying hypervariable signals across ChIPIATAC-seq samples. HyperChIP accounts for the mean-variability dependence intrinsic to sequencing count data by fitting an MVC, and it increases the statistical power by selecting a subset of genomic regions with low abundance of HVRs and LVRs and employing Winsorization in the parameter estimation procedure.

Throughout the study, we have strictly followed the criterion of separately handling proximal and distal regions in all hypervariable analyses. In summary, the variability structure associated with proximal regions differs from that of distal regions in many respects, including the underlying mean-variance trend, the distribution of HVRs along the range of mean signal intensities, and the global variability magnitude. In particular, the ChIP/ATAC-seq signal variability in distal regions is typically higher than that in proximal regions. Here, to provide a clear demonstration of this difference, we design a pipeline for comparing the global signal variability across a given set of ChIP/ATAC-seq samples between proximal and distal regions. Technically, we achieve it by comparing the variance ratio factors (denoted by $\gamma$ ) separately estimated from proximal and distal regions, while controlling for the MVC and the $d_{0}$ parameter (refer to the HyperChIP model formulated by equation (1) and (2)). In detail, we first process together proximal and distal regions in the normalization and MVC fitting procedures. Then, we set $d_{0}$ to positive infinity and apply the Winsorization framework (see Methods) separately to proximal and distal regions to estimate $\gamma$. In this way, the $\gamma$ estimate derived from proximal/distal regions represents the scaling factor of the MVC to reach the variance values observed at proximal/distal regions, and we can effectively compare the global signal variability between proximal and distal regions by comparing the corresponding $\gamma$ estimates. Applying this pipeline, we found that the $\gamma$ estimate derived from proximal 
regions was smaller than that from distal regions for each of the data sets in Table 1 (Fig. 7A). We further split the pan-cancer ATAC-seq data set into 23 small data sets corresponding to individual cancer types. Again, we observed a systematic increase in signal variability at distal regions compared to proximal regions (Fig. 7B). Together, these observations suggest the necessity of separately handling proximal and distal regions in hypervariable ChIP/ATAC-seq analysis.

\section{Methods}

\section{Data preprocessing}

For the data sets listed in Table 1, processing of all the associated RNA-seq and ChIP-seq samples started with raw sequencing reads. We first used Trim Galore (v0.4.4) to trim 3' ends of reads [56]. Resulting RNA-seq and ChIP-seq reads were then aligned to the hg19 reference genome by STAR (v2.5.1b) and Bowtie (v1.2.2), respectively $[57,58]$. To avoid artefacts from PCR amplification, we kept for each sample at most one read or read pair at each genomic location. The remaining reads or read pairs of each RNA-seq sample were then assigned to UCSC annotated genes [59] by htseq-count (v0.6.1p1) [60]. As for the (NSCLC) ATAC-seq samples, only alignment results for hg19 (BAM-formatted files) were available. Trimming of reads and removal of duplicates had already been done for these BAM files.

We next processed the read alignments of paired-end ChIP-seq samples. Specifically, each read pair was converted into a single read whose 5 ' end lay upstream of the associated DNA fragment center by $100 \mathrm{bp}$, with the fragment center inferred as the midpoint between the two 5 ' ends of the read pair. This processing guaranteed that the 5 ' ends of all the resulting reads lay upstream of the presumed protein binding sites by a fixed distance.

Accompanying input samples measuring background signals were available for all the ChIP-seq samples and were processed in the same way as were the ChIP-seq samples.

\section{Peak calling}

Peak calling for each ChIP-seq sample was performed against the corresponding input sample by using MACS (v1.4.2), with the parameters '--nomodel --shiftsize=100 --keep-dup=all' [61]. For the ATAC-seq samples, peaks were directly obtained from the corresponding study [18].

\section{Input matrices for normalization}

A count matrix and an occupancy matrix have been constructed for each data set by using MAnorm2_utils (v1.0.0) [24]. Rows and columns of both matrices corresponded 
to a list of genomic regions and the related ChIP/ATAC-seq samples, respectively. The count matrix recorded raw read counts. The occupancy matrix used binary variables to indicate whether each region was a peak region in each sample.

We invoked MAnorm2_utils with the parameters '--typical-bin-size $=X$--shiftsize $=Y$ --keep-dup=all --filter=blacklist' $(X$ was set to 2000 for the H3K27ac ChIP-seq data set and was set to 1000 for the ATAC-seq and Pol II ChIP-seq data sets; $Y$ was set to 100 for the H3K27ac and Pol II ChIP-seq data sets and was set to 0 for the ATACseq data set). The blacklisted regions for hg19 were obtained from Amemiya et al. [62].

For the two matrices associated with each data set, we then removed the genomic regions on sex chromosomes, since the patients were of different genders and our aim was to identify hypervariable signals relevant to cancer. We also classified the remaining regions into proximal and distal ones and accordingly split each matrix. Technically, each region with a distance less than $5 \mathrm{~kb}$ to any (UCSC annotated) transcription start site was defined to be a proximal region, and the other regions were defined to be distal ones. Unless otherwise stated, all downstream analyses (e.g., normalization and hypervariable analysis) targeting an individual data set have separately handled proximal and distal regions.

\section{Normalization}

We detail here the method used in the study for deriving normalized $\log _{2}$ read counts as input data of HyperChIP. Given a count matrix and an occupancy matrix, suppose $K_{i j}$ is the read count at region $i$ in sample $j$ and that $O_{i j}$ indicates the associated occupancy status. Each $O_{i j}$ takes a value of 0 or 1 , and $O_{i j}=1$ indicates region $i$ is a peak region in sample $j$ (or region $i$ is occupied by sample $j$ ). Define $Y_{i j}=$ $\log _{2}\left(K_{i j}+0.5\right)$.

To normalize the samples, we first construct a pseudo-reference profile as normalization baseline, which is defined by

$$
P_{i}=\left\{\begin{array}{cl}
\frac{\sum_{j} O_{i j} Y_{i j}}{\sum_{j} O_{i j}} & \sum_{j} O_{i j}>0, \\
0 & \sum_{j} O_{i j}=0 .
\end{array}\right.
$$

The above definition is for notational convenience. In fact, the $P_{i}$ with which the associated $\sum_{j} O_{i j}$ are 0 will never be used in the subsequent normalization procedures.

We next repeatedly normalize each sample against the baseline. Technically, the normalization of sample $j$ is accomplished by applying a linear transformation to all the corresponding $Y_{i j}$. Formally, let $X_{i j}=\alpha_{j}+\beta_{j} Y_{i j}$ be normalized $\log _{2}$ read counts, where $\alpha_{j}$ and $\beta_{j}$ are coefficients to be determined. For notational simplicity, we 
further define $\mathrm{M}$ and $\mathrm{A}$ values as $M_{i j}=X_{i j}-P_{i}$ and $A_{i j}=\left(P_{i}+X_{i j}\right) / 2$, respectively, and define $n_{j}=\sum_{i} O_{i j}$ to be the total number of regions that are occupied by sample $j$. The two coefficients are determined by imposing the following two constraints:

$$
\begin{gathered}
\sum_{i: O_{i j}=1} M_{i j}=0, \\
\sum_{i: O_{i j}=1}\left(M_{i j}-\frac{\sum_{i^{\prime}: o_{i^{\prime} j}=1} M_{i^{\prime} j}}{n_{j}}\right)\left(A_{i j}-\frac{\sum_{i^{\prime}: o_{i^{\prime} j}=1} A_{i^{\prime} j}}{n_{j}}\right)=0 .
\end{gathered}
$$

Intrinsically, the above two constraints are for simultaneously removing the global signal difference and $\mathrm{M}-\mathrm{A}$ trend at common peak regions of sample $j$ and the pseudo-reference profile. Solutions for $\alpha_{j}$ and $\beta_{j}$ are given by

$$
\begin{gathered}
\alpha_{j}=\frac{\sum_{i: o_{i j}=1} P_{i}-\beta_{j} \sum_{i: o_{i j}=1} Y_{i j}}{n_{j}}, \\
\beta_{j}=\sqrt{\frac{\sum_{i: o_{i j}=1}\left(P_{i}-\frac{\sum_{i^{\prime}: o_{i^{\prime} j}=1} P_{i^{\prime}}}{n_{j}}\right)^{2}}{\sum_{i: o_{i j}=1}\left(Y_{i j}-\frac{\sum_{i^{\prime}: o_{i^{\prime} j}=1} Y_{i^{\prime} j}}{n_{j}}\right)^{2}}} .
\end{gathered}
$$

\section{Parameter estimation for the HyperChIP model}

The whole model formulation of HyperChIP as well as the associated hypothesis testing procedure is given by the equations (1-3). Here, we detail the method for estimating $d_{0}$ and $\gamma$.

We first explain the application of the Winsorization technique. The idea is similar to robust limma [63] and we apply moment estimation to Winsorized log scaled variances. Formally, let $z_{i}=\log \frac{\hat{t}_{i}}{f\left(\widehat{\mu}_{i}\right)}$ be the log scaled variance associated with region $i$. Set $p_{l}$ and $p_{u}$ to two small values representing the maximum proportions of outliers allowed in the lower and upper tails of all $z_{i}$, respectively (in the study, $p_{l}=0.01$ and $p_{u}=0.1$ were always used). Let $q_{l}$ and $q_{u}$ be the corresponding lower and upper sample quantiles of $z_{i}$, respectively. Then, Winsorized $z_{i}$ are defined by

$$
\operatorname{win}\left(z_{i} ; p_{l}, p_{u}\right)=\left\{\begin{array}{lr}
q_{l} & z_{i} \leq q_{l}, \\
z_{i} & q_{l}<z_{i}<q_{u}, \\
q_{u} & z_{i} \geq q_{u} .
\end{array}\right.
$$

Based on the model, it approximately follows that

$$
\operatorname{win}\left(z_{i} ; p_{l}, p_{u}\right) \sim \log \gamma+\log \left[\operatorname{win}\left(F_{m-1, d_{0}} ; p_{l}, p_{u}\right)\right] \text {. }
$$

Note that, in contrast with the Winsorization of $z_{i}$, the Winsorization of $F_{m-1, d_{0}}$ in the above formula refers to squeezing certain proportions at two tails of the probability 
density function towards the corresponding theoretical quantiles, which results in a mixture of a continuous distribution with a bounded support domain and two point masses at the edges.

We next apply a moment estimation approach. It follows from (10) that

$$
\begin{gathered}
E\left[\operatorname{win}\left(z_{i} ; p_{l}, p_{u}\right)\right]=\log \gamma+E\left\{\log \left[\operatorname{win}\left(F_{m-1, d_{0}} ; p_{l}, p_{u}\right)\right]\right\}, \\
\operatorname{var}\left[\operatorname{win}\left(z_{i} ; p_{l}, p_{u}\right)\right]=\operatorname{var}\left\{\log \left[\operatorname{win}\left(F_{m-1, d_{0}} ; p_{l}, p_{u}\right)\right]\right\} .
\end{gathered}
$$

The approach is to first solve (12) for $d_{0}$ and then solve (11) for $\gamma$. Technically, given $d_{0}$, the expectation and variance on the right-hand sides of (11) and (12) respectively are calculated by turning to Gauss-Legendre quadrature [64], and (12) is solved by applying the bisection method.

Besides using Winsorization, HyperChIP also makes a selection of low-intensity regions for parameter estimation. By default, the above Winsorization process is only applied to the $z_{i}$ of the $10 \%$ of regions with the smallest $\hat{\mu}_{i}$.

\section{Applying other methods for ranking HVRs}

For the methods that do not consider the mean-variability dependence, the associated variability statistics, including observed variance, MAD, and IQR, were calculated from the same normalized $\log _{2}$ read counts as used by HyperChIP. For the min-rank method, it was applied to the same normalized data as well. For the method that uses a moderately large offset when applying a $\log _{2}$ transformation, we followed the computational pipeline presented in the original study [17]. More specifically, we first derived $\log _{2}-\mathrm{CPM}$ values by using the $\mathrm{cpm}$ function of the edgeR package [65], with setting log=TRUE and prior. count to a large value (5 or 10 in this study). We then applied quantile normalization to the $\log _{2}$-CPM values and calculated the observed variance associated with each region. Finally, these variances were used to rank HVRs.

\section{Identifying HVGs}

We identified HVGs separately for each data set, by applying limma-trend $[23,66]$ to the corresponding RNA-seq data. Technically, we first converted RNA-seq read counts into $\log _{2}$-CPM values by using the calcNormFactors and cpm functions of the edgeR package, with log=TRUE for the latter. Then, the standard limma pipeline [67] was applied to the $\log _{2}$-CPM values, with trend=TRUE for the eBayes function (note that the design matrix contained only an intercept variable). The ratios of sample variances to prior variances were then used as key statistics for identifying HVGs, and we applied a one-sided $p$-value cutoff of 0.05 .

\section{Classification of the NSCLC ATAC-seq samples}

Each classification of the samples was performed by using a set of top-ranked HVRs 
selected by some method as features. We first separately scaled the normalized $\log _{2}$ read counts associated with each HVR for all the methods but the one applying a large offset for $\log _{2}$ transformation. According to the analysis pipeline presented in the original study [17], we directly used the normalized data of the large-offset method for the subsequent clustering analysis. Then, we calculated the Euclidean distance between each pair of samples and performed a hierarchical clustering by using the hclust function of the R software [68], with method="ward. D". The resulting hierarchical tree was then cut into two branches, and we assessed the consistency between this classification and the true labels (i.e., LUAD or LUSC) by calculating the ARI, which was achieved by using the adjustedRandlndex function of the mclust package [69].

\section{Biological interpretation of the significant HVRs and LVRs identified for the LUAD H3K27ac ChIP-seq data set}

For the GO enrichment analysis, GO terms for biological processes were obtained from the Molecular Signatures Database (MSigDB) [70]. Enrichments of GO terms were assessed by performing Fisher's exact tests, with all the proximal H3K27ac peak region-associated genes as background.

For the histopathological labels of the LUAD patients, we first made a classification based on the most prevalent histologic pattern of each patient, which was lepidic, papillary, acinar, or solid [71]. According to the original study [6], we then specified the histopathological labels of the lepidic-prevalent and solid-prevalent patients as low-risk and high-risk, respectively, and the remaining patients were considered median-risk. Finally, we ranked all patients based on their risk levels and quantified the Spearman correlations of H3K27ac ChIP-seq signals in different regions with the risk levels.

We also separately performed a regression of the survival time of the patients on the H3K27ac ChIP-seq signals in each region, by fitting a Cox proportional hazards model [41]. Technically, this was achieved by using the CoxPHFitter function of the lifelines package [72], and we used the (two-sided) $p$-value associated with each model to evaluate the prognostic association of the corresponding region. For the hierarchical clustering of the patients, we followed the same pipeline as used for classifying the NSCLC ATAC-seq samples, except that the proximal and distal HVRs were used together as features. Testing the survival difference between the resulting two sub-groups was achieved by using the survdiff function of the survival package [73].

The phastCons scores for assessing the sequence conservation of different regions were obtained from the phastCons100way.UCSC.hg19 package [36]. Each base with a phastCons score over 0.9 was considered conserved. 


\section{Identifying ATAC-seq QTLs}

For the NSCLC ATAC-seq data set, among the germline SNPs located within peak regions, we have identified QTLs whose different genotypes were associated with significantly differential ATAC-seq signals in the enclosing peak region. For this analysis, we first noticed that all the SNPs were associated with exactly two different genotypes across the individuals, referred to as reference and alternative genotypes. To increase the statistical power, we filtered out the SNPs whose reference or alternative genotype was associated with less than 5 individuals. Then, we performed a two-sample $t$-test between the ATAC-seq signals (i.e., the normalized $\log _{2}$ read counts used by HyperChIP) associated with the two genotypes of each SNP. Finally, the SNPs with a $\mathrm{BH}$-adjusted $p$-value less than 0.1 were considered as significant QTLs.

\section{Analysis of the pan-cancer ATAC-seq data set}

The standard HyperChIP pipeline was applied to the whole data set. For the input data, the count matrix was downloaded from the corresponding TCGA page (https://gdc.cancer.gov/about-data/publications/ATACseq-AWG), and the occupancy matrix was determined by considering the genomic regions with $>50$ read counts as peak regions.

For the t-SNE analysis, we first separately scaled the ATAC-seq signals at each HVR. Formally, suppose $X_{i j}$ is the normalized $\log _{2}$ read count at HVR $i$ in the ATAC-seq sample $j$. Let $X_{i}=\left(X_{i 1}, X_{i 2}, \cdots\right)$ be the vector of normalized $\log _{2}$ read counts at HVR $i$ in all the samples. We define

$$
Z_{i j}=\frac{X_{i j}-\operatorname{mean}\left(X_{i .}\right)}{\operatorname{sd}\left(X_{i}\right)}
$$

where mean and sd refer to sample mean and sample standard deviation, respectively. Let $Z_{\cdot j}=\left(Z_{1 j}, Z_{2 j}, \cdots\right)$ be the scaled ATAC-seq signals at all the identified HVRs in sample $j$. We then performed a principal component analysis by using $Z_{\cdot j}$ as the features of sample $j$. Finally, the t-SNE routine implemented in the Rtsne package [74] was applied to the first 50 principal components, which led to the two-dimensional t-SNE plot.

For the identification of class-specific regulators, we first performed a motifscanning analysis by using the matchMotifs function of the motifmatchr package [46] with default settings. Let $I_{m i}$ indicate whether HVR $i$ contains an instance of motif $m$. Each $I_{m i}$ takes a value of 0 or 1 . Then, we defined the activity score of each motif $m$ in each sample $j$ as 


$$
S_{m j}=\frac{\sum_{i} I_{m i}\left(Z_{i j}-\operatorname{mean}\left(Z_{\cdot j}\right)\right)}{\sum_{i} I_{m i}},
$$

where the sum operators are applied to all the HVRs and the subtraction is for adjusting for sample-specific biases. Finally, for each of the four classes of cancer types, we performed a two-sample $t$-test for each motif to compare its activity scores between the samples belonging to the class and the other samples. Supplementary Table 4 gives the top 15 motifs identified for each class.

\section{Availability of software and analysis results}

The whole HyperChIP model as well as the MA normalization method based on a pseudo-reference profile has been implemented and incorporated into the MAnorm2 package (v1.1.0), which is provided as Supplementary Software and can also be accessed via https://github.com/tushiqi/MAnorm2. Supplementary Table 5 gives the significant HVRs and LVRs (BH-adjusted $p$-value $<0.1)$ identified for each of the data sets in Table 1.

\section{Competing interest statement}

The authors declare no competing interests.

\section{Acknowledgments}

This work was supported by the National Basic Research Program of China (2018YFA0107602 and 2018YFA0800203), the National Natural Science Foundation of China (31871280 and 31701140), and the Strategic Priority Research Program of Chinese Academy of Sciences (XDB38040100).

\section{Author contributions}

S.T. and Z.S. conceived the study; H.C. and S.T. developed the algorithms and analyzed the data; S.T. and Z.S. jointly supervised the study; H.C., S.T., and Z.S. wrote the manuscript with contributions from all the other authors.

\section{References}

1. Mardis, E.R., ChIP-seq: welcome to the new frontier. Nat Methods, 2007. 4(8): p. 613-4.

2. Park, P.J., ChIP-seq: advantages and challenges of a maturing technology. Nat Rev Genet, 2009. 10(10): p. 669-80. 
3. Buenrostro, J.D., et al., Transposition of native chromatin for fast and sensitive epigenomic profiling of open chromatin, DNA-binding proteins and nucleosome position. Nat Methods, 2013. 10(12): p. 1213-8.

4. Gifford, C.A., et al., Transcriptional and epigenetic dynamics during specification of human embryonic stem cells. Cell, 2013. 153(5): p. 1149-63.

5. Lara-Astiaso, D., et al., Immunogenetics. Chromatin state dynamics during blood formation. Science, 2014. 345(6199): p. 943-9.

6. Yuan, C., et al., A systematic dissection of the epigenomic heterogeneity of lung adenocarcinoma reveals two different subclasses with distinct prognosis and core regulatory networks. Genome Biol, 2021. 22(1): p. 156.

7. Steinhauser, S., et al., A comprehensive comparison of tools for differential ChIP-seq analysis. Brief Bioinform, 2016. 17(6): p. 953-966.

8. Tu, S. and Z. Shao, An introduction to computational tools for differential binding analysis with ChIP-seq data. Quantitative Biology, 2017. 5(3): p. 226-235.

9. Stelloo, S., et al., Integrative epigenetic taxonomy of primary prostate cancer. Nat Commun, 2018. 9(1): p. 4900.

10. Yi, G.Q., et al., Chromatin-Based Classification of Genetically Heterogeneous AMLs into Two Distinct Subtypes with Diverse Stemness Phenotypes. Cell Reports, 2019. 26(4): p. 1059-+.

11. Stegle, O., S.A. Teichmann, and J.C. Marioni, Computational and analytical challenges in single-cell transcriptomics. Nat Rev Genet, 2015. 16(3): p. 133-45.

12. Kim, N., et al., Single-cell RNA sequencing demonstrates the molecular and cellular reprogramming of metastatic lung adenocarcinoma. Nat Commun, 2020. 11(1): p. 2285.

13. Maynard, A., et al., Therapy-Induced Evolution of Human Lung Cancer Revealed by Single-Cell RNA Sequencing. Cell, 2020. 182(5): p. 1232-1251 e22.

14. Brennecke, P., et al., Accounting for technical noise in single-cell RNA-seq experiments. Nat Methods, 2013. 10(11): p. 1093-5.

15. Vallejos, C.A., J.C. Marioni, and S. Richardson, BASiCS: Bayesian Analysis of Single-Cell Sequencing Data. PLoS Comput Biol, 2015. 11(6): p. e1004333.

16. Chen, H.I., et al., Detection of high variability in gene expression from single-cell RNA-seq profiling. BMC Genomics, 2016. 17 Suppl 7: p. 508.

17. Corces, M.R., et al., The chromatin accessibility landscape of primary human cancers. Science, 2018. 362(6413).

18. Wang, Z., et al., The Open Chromatin Landscape of Non-Small Cell Lung Carcinoma. Cancer Res, 2019. 79(19): p. 4840-4854.

19. Cejas, P., et al., Chromatin immunoprecipitation from fixed clinical tissues reveals tumorspecific enhancer profiles. Nat Med, 2016. 22(6): p. 685-91.

20. Lomberk, G., et al., Distinct epigenetic landscapes underlie the pathobiology of pancreatic cancer subtypes. Nat Commun, 2018. 9(1): p. 1978.

21. Raisner, R., et al., Super-enhancer acquisition drives oncogene expression in triple negative breast cancer. PLoS One, 2020. 15(6): p. e0235343.

22. Iyyanki, T., et al., Subtype-associated epigenomic landscape and 3D genome structure in bladder cancer. Genome Biol, 2021. 22(1): p. 105.

23. Law, C.W., et al., voom: Precision weights unlock linear model analysis tools for RNA-seq read counts. Genome Biol, 2014. 15(2): p. R29. 
24. Tu, S., et al., MAnorm2 for quantitatively comparing groups of ChIP-seq samples. Genome Res, 2021. 31(1): p. 131-145.

25. Tukey, J.W., The Future of Data Analysis. The Annals of Mathematical Statistics, 1962. 33(1): p. 1-67, 67.

26. Benjamini, Y. and Y. Hochberg, Controlling the False Discovery Rate - a Practical and Powerful Approach To Multiple Testing. Journal of the Royal Statistical Society Series B-Statistical Methodology, 1995. 57(1): p. 289-300.

27. Ernst, J., et al., Mapping and analysis of chromatin state dynamics in nine human cell types. Nature, 2011. 473(7345): p. 43-9.

28. Kasowski, M., et al., Extensive variation in chromatin states across humans. Science, 2013. 342(6159): p. 750-2.

29. Heinz, S., et al., The selection and function of cell type-specific enhancers. Nat Rev Mol Cell Biol, 2015. 16(3): p. 144-54.

30. Ramsey, M.R., et al., FGFR2 signaling underlies $p 63$ oncogenic function in squamous cell carcinoma. J Clin Invest, 2013. 123(8): p. 3525-38.

31. Yi, M., et al., TP63 links chromatin remodeling and enhancer reprogramming to epidermal differentiation and squamous cell carcinoma development. Cell Mol Life Sci, 2020. 77(21): p. 4325-4346.

32. Suzuki, A., et al., Aberrant transcriptional regulations in cancers: genome, transcriptome and epigenome analysis of lung adenocarcinoma cell lines. Nucleic Acids Res, 2014. 42(22): p. 13557-72.

33. Loader, C., locfit: Local Regression, Likelihood and Density Estimation. 2020.

34. Hubert, L. and P. Arabie, Comparing partitions. Journal of Classification, 1985. 2(1): p. 193218.

35. Love, M.I., W. Huber, and S. Anders, Moderated estimation of fold change and dispersion for RNA-seq data with DESeq2. Genome Biol, 2014. 15(12): p. 550.

36. Siepel, A., et al., Evolutionarily conserved elements in vertebrate, insect, worm, and yeast genomes. Genome Res, 2005. 15(8): p. 1034-50.

37. Whitsett, J.A., S.E. Wert, and T.E. Weaver, Alveolar surfactant homeostasis and the pathogenesis of pulmonary disease. Annu Rev Med, 2010. 61: p. 105-19.

38. Cheung, W.K. and D.X. Nguyen, Lineage factors and differentiation states in lung cancer progression. Oncogene, 2015. 34(47): p. 5771-80.

39. Sainz de Aja, J., A.F.M. Dost, and C.F. Kim, Alveolar progenitor cells and the origin of lung cancer. J Intern Med, 2021. 289(5): p. 629-635.

40. LaFave, L.M., et al., Epigenomic State Transitions Characterize Tumor Progression in Mouse Lung Adenocarcinoma. Cancer Cell, 2020. 38(2): p. 212-228 e13.

41. Andersen, P.K. and R.D. Gill, Cox Regression-Model for Counting-Processes - a Large Sample Study. Annals of Statistics, 1982. 10(4): p. 1100-1120.

42. Grubert, F., et al., Genetic Control of Chromatin States in Humans Involves Local and Distal Chromosomal Interactions. Cell, 2015. 162(5): p. 1051-65.

43. Van der Maaten, L. and G. Hinton, Visualizing data using t-SNE. Journal of machine learning research, 2008. 9(11).

44. Tabibu, S., P.K. Vinod, and C.V. Jawahar, Pan-Renal Cell Carcinoma classification and survival prediction from histopathology images using deep learning. Scientific Reports, 2019. 9. 
45. Fornes, O., et al., JASPAR 2020: update of the open-access database of transcription factor binding profiles. Nucleic Acids Res, 2020. 48(D1): p. D87-D92.

46. Schep, A., motifmatchr: Fast Motif Matching in R. 2019.

47. Babeu, J.P. and F. Boudreau, Hepatocyte nuclear factor 4-alpha involvement in liver and intestinal inflammatory networks. World J Gastroenterol, 2014. 20(1): p. 22-30.

48. Yeh, M.M., D.E. Bosch, and S.S. Daoud, Role of hepatocyte nuclear factor 4-alpha in gastrointestinal and liver diseases. World J Gastroenterol, 2019. 25(30): p. 4074-4091.

49. Tang, Z., et al., GEPIA: a web server for cancer and normal gene expression profiling and interactive analyses. Nucleic Acids Res, 2017. 45(W1): p. W98-W102.

50. Real Hernandez, L.M., et al., Berry Phenolic Compounds Increase Expression of Hepatocyte Nuclear Factor-1alpha (HNF-1alpha) in Caco-2 and Normal Colon Cells Due to High Affinities with Transcription and Dimerization Domains of HNF-1alpha. PLoS One, 2015. 10(9): p. e0138768.

51. Rothenberg, S.M. and L.W. Ellisen, The molecular pathogenesis of head and neck squamous cell carcinoma. J Clin Invest, 2012. 122(6): p. 1951-7.

52. Cao, B.J., et al., Use of four genes in exosomes as biomarkers for the identification of lung adenocarcinoma and lung squamous cell carcinoma. Oncology Letters, 2021. 21(4).

53. Cancer Genome Atlas Research, N., et al., Integrated genomic characterization of oesophageal carcinoma. Nature, 2017. 541(7636): p. 169-175.

54. Zhang, D., D.C. Zeldin, and P.J. Blackshear, Regulatory factor X4 variant 3: a transcription factor involved in brain development and disease. J Neurosci Res, 2007. 85(16): p. 3515-22.

55. Jeong, H.Y., et al., High expression of RFX4 is associated with tumor progression and poor prognosis in patients with glioblastoma. International Journal of Neuroscience, 2021. 131(1): p. 7-14.

56. Martin, M., Cutadapt removes adapter sequences from high-throughput sequencing reads. 2011, 2011. 17(1): p. 3.

57. Dobin, A., et al., STAR: ultrafast universal RNA-seq aligner. Bioinformatics, 2013. 29(1): p. 1521.

58. Langmead, B., et al., Ultrafast and memory-efficient alignment of short DNA sequences to the human genome. Genome Biol, 2009. 10(3): p. R25.

59. Karolchik, D., et al., The UCSC Table Browser data retrieval tool. Nucleic Acids Res, 2004. 32(Database issue): p. D493-6.

60. Anders, S., P.T. Pyl, and W. Huber, HTSeq--a Python framework to work with high-throughput sequencing data. Bioinformatics, 2015. 31(2): p. 166-9.

61. Zhang, Y., et al., Model-based analysis of ChIP-Seq (MACS). Genome Biol, 2008. 9(9): p. R137.

62. Amemiya, H.M., A. Kundaje, and A.P. Boyle, The ENCODE Blacklist: Identification of Problematic Regions of the Genome. Sci Rep, 2019. 9(1): p. 9354.

63. Phipson, B., et al., Robust Hyperparameter Estimation Protects against Hypervariable Genes and Improves Power To Detect Differential Expression. Ann Appl Stat, 2016. 10(2): p. 946-963.

64. Smyth, G.K., Numerical integration. Encyclopedia of Biostatistics, 2005: p. 3088-3095.

65. Robinson, M.D., D.J. McCarthy, and G.K. Smyth, edgeR: a Bioconductor package for differential expression analysis of digital gene expression data. Bioinformatics, 2010. 26(1): p. 139-40.

66. Sartor, M.A., et al., Intensity-based hierarchical Bayes method improves testing for 
bioRxiv preprint doi: https://doi.org/10.1101/2021.07.27.453915; this version posted July 27, 2021. The copyright holder for this preprint

(which was not certified by peer review) is the author/funder, who has granted bioRxiv a license to display the preprint in perpetuity. It is made available under aCC-BY-NC-ND 4.0 International license.

differentially expressed genes in microarray experiments. BMC Bioinformatics, 2006. 7: p.

538.

67. Smyth, G.K., Linear models and empirical bayes methods for assessing differential expression in microarray experiments. Stat Appl Genet Mol Biol, 2004. 3: p. Article3.

68. R Core Team, R: A Language and Environment for Statistical Computing. 2020, R Foundation for Statistical Computing: Vienna, Austria.

69. Scrucca, L., et al., mclust 5: clustering, classification and density estimation using Gaussian finite mixture models. The R Journal, 2016. 8(1): p. 289-317.

70. Liberzon, A., et al., Molecular signatures database (MSigDB) 3.0. Bioinformatics, 2011. 27(12): p. 1739-40.

71. Tavernari, D., et al., Nongenetic Evolution Drives Lung Adenocarcinoma Spatial Heterogeneity and Progression. Cancer Discov, 2021.

72. Davidson-Pilon, C. lifelines 0.26.0. 2021; Available from: https://pypi.org/project/lifelines/.

73. Therneau, T.M., A Package for Survival Analysis in S. 2015.

74. Krijthe, J.H., Rtsne: T-Distributed Stochastic Neighbor Embedding using a Barnes-Hut Implementation. 2015. 


\section{Tables}

\begin{tabular}{cccc}
\hline Data set & Biological context & Cohort size & Source \\
\hline H3K27ac ChIP-seq & $\begin{array}{c}\text { Tumor tissues } \\
\text { of LUAD patients }\end{array}$ & 36 & Yuan et al. [6] \\
ATAC-seq & $\begin{array}{c}\text { Tumor tissues } \\
\text { of NSCLC patients }\end{array}$ & 34 & Wang et al. [18] \\
Pol II ChIP-seq & $\begin{array}{c}\text { Cancer cell lines } \\
\text { derived from LUAD patients }\end{array}$ & 26 & Suzuki et al. [32] \\
\hline
\end{tabular}

Table 1. Data sets used for evaluating the performance of HyperChIP. Each data set was comprised of ChIP/ATAC-seq profiles of tens of different cancer patients. LUAD, lung adenocarcinoma; NSCLC, non-small cell lung carcinoma. 


\begin{tabular}{|c|c|c|c|c|}
\hline \multirow{2}{*}{$\begin{array}{l}\text { Parameter } \\
\text { estimation }\end{array}$} & \multirow{2}{*}{ Data set } & \multicolumn{3}{|c|}{ Number of significant proximal HVRs } \\
\hline & & 0.01 & 0.05 & 0.1 \\
\hline \multirow{3}{*}{ Original } & H3K27ac ChIP-seq & 0 & 2 & 2 \\
\hline & ATAC-seq & 5 & 8 & 10 \\
\hline & Pol II ChIP-seq & 0 & 0 & 0 \\
\hline \multirow{3}{*}{$\begin{array}{l}\text { Lower } 10 \%+ \\
\text { Winsorization }\end{array}$} & H3K27ac ChIP-seq & 237 & 789 & 1442 \\
\hline & ATAC-seq & 1050 & 2171 & 3197 \\
\hline & Pol II ChIP-seq & 303 & 990 & 1546 \\
\hline
\end{tabular}

Table 2. Numbers of significant proximal HVRs identified by applying different parameter estimation methods with various cutoffs of the BH-adjusted $p$-value. The original method refers to the moment matching method used by MAnorm2, and the other method corresponds to the default settings of HyperChIP. 


\begin{tabular}{|c|c|c|c|c|}
\hline \multirow{2}{*}{$\begin{array}{l}\text { Parameter } \\
\text { estimation }\end{array}$} & \multirow{2}{*}{ Data set } & \multicolumn{3}{|c|}{ Number of significant distal HVRs } \\
\hline & & 0.01 & 0.05 & 0.1 \\
\hline \multirow{3}{*}{ Original } & H3K27ac ChIP-seq & 2 & 8 & 12 \\
\hline & ATAC-seq & 0 & 3 & 4 \\
\hline & Pol II ChIP-seq & 0 & 0 & 0 \\
\hline \multirow{3}{*}{$\begin{array}{l}\text { Lower } 10 \%+ \\
\text { Winsorization }\end{array}$} & H3K27ac ChIP-seq & 289 & 1372 & 2621 \\
\hline & ATAC-seq & 3124 & 6830 & 10234 \\
\hline & Pol II ChIP-seq & 602 & 1400 & 2122 \\
\hline
\end{tabular}

Table 3. Numbers of significant distal HVRs identified by applying different parameter estimation methods with various cutoffs of the $\mathrm{BH}$-adjusted $p$-value. 


\section{Figure Legends}

Figure 1. Using scaled variances to rank genomic regions. (A) Scatter plots showing mean-variance trends associated with different data sets. Variance is shown at the $\log _{10}$ scale. Red lines depict the corresponding MVCs. Red points mark the 1000 regions with the largest scaled variances. (B) A scatter plot of $\log _{10}$ scaled variances against observed mean signal intensities. (C, D) For each data set in Table 1 , the TDP among top-ranked proximal regions is plotted against the number of topranked proximal regions for each method. MAD, median absolute deviation; IQR, interquartile range.

Figure 2. Evaluating classifications of samples that are based on top-ranked HVRs identified by different methods. (A, B) For each method applied to the ATAC-seq data set, the ARI is plotted against the number of top-ranked HVRs that are used to classify the ATAC-seq samples. Each ARI value assesses the consistency between a classification and the true labels of the samples. Proximal and distal regions are separately analyzed and ranked. (C) Box plots for the ARI values resulting from using a wide range of numbers of top-ranked HVRs to classify the samples. For each method, the sequence of numbers starts with 50 and ends with 5000 , with an increment of 50 .

\section{Figure 3. Selecting a subset of genomic regions and using Winsorization for} parameter estimation. (A) For the H3K27ac ChIP-seq data set, bar plots showing the distributions of top-ranked proximal/distal HVRs along the range of mean intensities. Proximal and distal regions have been separately divided into 10 equallysized groups based on the observed mean signal intensities. (B) Scatter plots of $\log _{10}$ scaled variances against mean intensities of proximal/distal regions. Top-ranked HVRs and LVRs are separately highlighted. (C) Bar plots showing the distributions of top-ranked proximal/distal LVRs along the range of mean intensities. (D) $d_{0}$ estimates resulting from different parameter estimation methods. The original method refers to the moment matching method employed by MAnorm2, which does not apply a subset selection and Winsorization. The method labeled as "lower $10 \%$ " uses only the $10 \%$ of regions having the smallest mean intensities for parameter estimation.

\section{Figure 4. HyperChIP is associated with an improved statistical power for} identifying HVRs. (A, B) For the $10 \%$ of genomic regions having the smallest mean intensities, plotting sample quantiles of the scaled variances against theoretical quantiles of the null distribution inferred by $(A)$ the original method or $(B)$ HyperChIP (i.e., the method labeled as "lower 10\% + Winsorization"). (C, D) The overall 
distributions of (one-tailed) $p$-values resulting from (C) the original method or (D) HyperChIP.

Figure 5. Biological interpretation of HVRs and LVRs. (A) For each gene set, the number of significant GO terms is plotted against the $p$-value cutoff used in the GO enrichment analysis. Significant proximal HVRs/LVRs used here are defined based on the LUAD H3K27ac ChIP-seq data set. (B) Example GO terms enriched from the genes linked with the significant proximal LVRs. Supplementary Table 1 gives a full list of the top $20 \mathrm{GO}$ terms. (C, D) Box plots showing the fractions of conserved bases in different genomic regions. Here, the conserved bases are defined as those with a phastCons score over 0.9 . Red dotted lines indicate the genome-wide fraction. (E) Example GO terms enriched from the genes linked with the significant proximal HVRs. Supplementary Table 2 gives a full list of the top 20 GO terms. (F, G) Box plots showing the correlations of $\mathrm{H} 3 \mathrm{~K} 27 \mathrm{ac}$ levels in different regions with the clinical stage of LUAD progression. Here, the correlations are measured by the absolute value of the Spearman correlation coefficient. (H) Significant proximal HVRs defined for the NSCLC ATAC-seq data set are enriched with somatic SNVs. We have performed 1,000 times of random simulation. In each time, a set of proximal peak regions matching the number of the HVRs has been randomly selected. (I) Significant distal HVRs are enriched with somatic SNVs as well.

\section{Figure 6. Applying HyperChIP to a TCGA pan-cancer ATAC-seq data set. (A)}

Two-dimensional t-SNE plot of all patients based on their ATAC-seq profiles of tumor tissues. The patients are colored by cancer types. See Supplementary Table 3 for the full names of the involved cancer types. (B) The distribution of the patients belonging in the SC class. (C) The distribution of the patients belonging in the DIAD class. (D) The distribution of all ESCA patients, comprised of 12 ESSC and 6 ESAD cases. (E) Heat map showing the TF activity scores of the top 15 binding motifs identified for each class of cancer types. (F) Plotting the $t$-statistics of all motifs against their rankings in the identification of DIAD class-specific TFs. (G) Mapping the TF activity scores of the HNF4A motif to the t-SNE plot. (H) Mapping the expression levels of the HNF4A gene (calculated from the corresponding RNA-seq samples) to the t-SNE plot. (I) Box plots showing the expression of HNF4A in a larger TCGA cohort of $(7,183)$ patients. The expression data are accessed via the online tool GEPIA, in which ESSC and ESAD patients are both labeled ESCA and cannot be distinguished from each other. The cancer types are sorted by the median expression of HNF4A in tumors. For each cancer type, the significance of differential expression is determined by performing a $t$-test with a $p$-value cutoff of 0.01 and a fold change cutoff of 2. TPM, transcripts per million. (J) Plotting the $t$-statistics of all motifs against 
their rankings in the identification of SC class-specific TFs. (K) Mapping the TF activity scores of the TP63.1 motif to the t-SNE plot. (L) Mapping the expression levels of the TP63 gene to the t-SNE plot. (M) Box plots showing the expression of TP63 in the larger TCGA cohort.

Figure 7. Comparing the global ChIPIATAC-seq signal variability in proximal genomic regions with that in distal regions. (A) Comparing the $\gamma$ estimate derived from proximal regions with that from distal regions for each of the data sets in Table 1. (B) Comparing $\gamma$ estimates separately derived from proximal and distal regions for the pan-cancer ATAC-seq data set, which have been split into 23 small data sets corresponding to individual cancer types. 
Ei ur bio Rxiv preprint doi: https://doi.org/10.1101/2021.07.27.453915; this version posted July 27, 2021. The copyright holder for this preprint - IgUrentich was not certified by peer review) is the author/funder, who has granted bioRxiv a license to display the preprint in perpetuity. It is made available under aCC-BY-NC-ND 4.0 International license.

A

H3K27ac: proximal regions

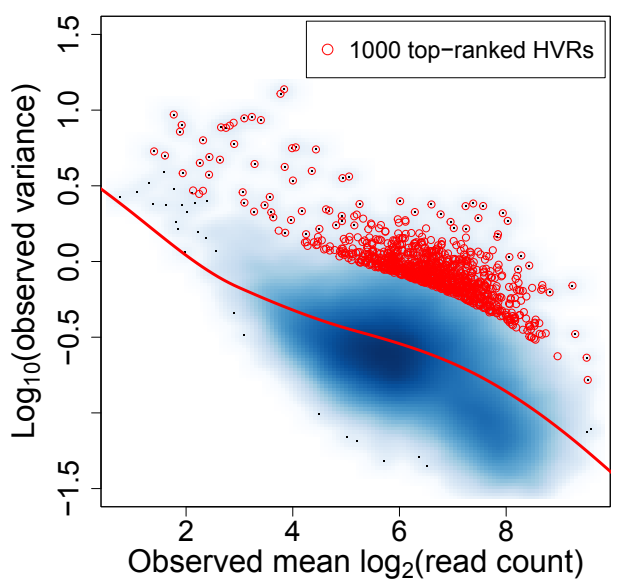

H3K27ac

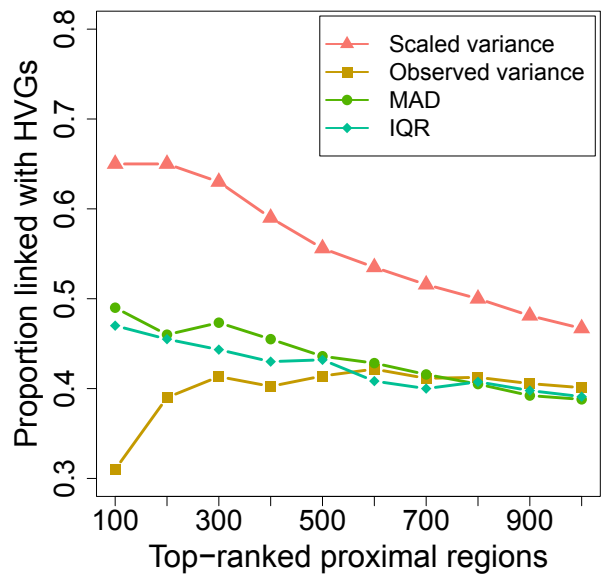

$\mathrm{D}$

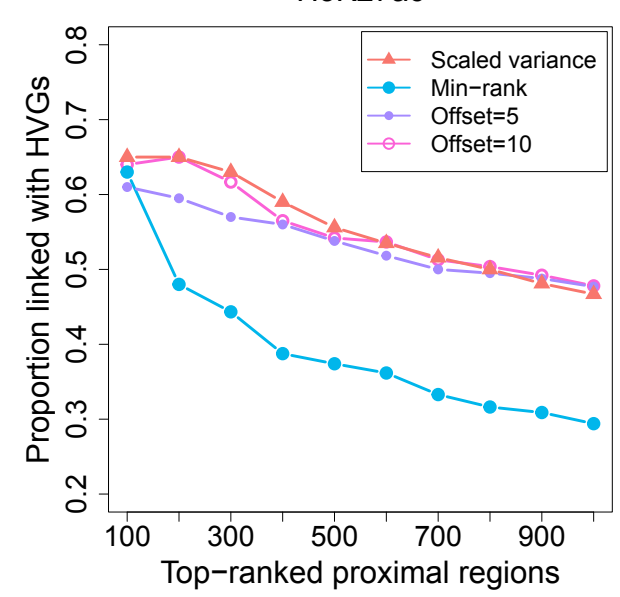

B

ATAC-seq: proximal regions

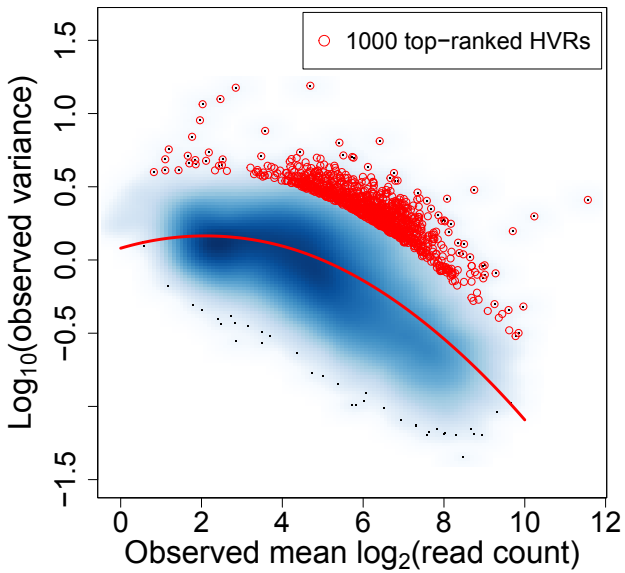

ATAC-seq

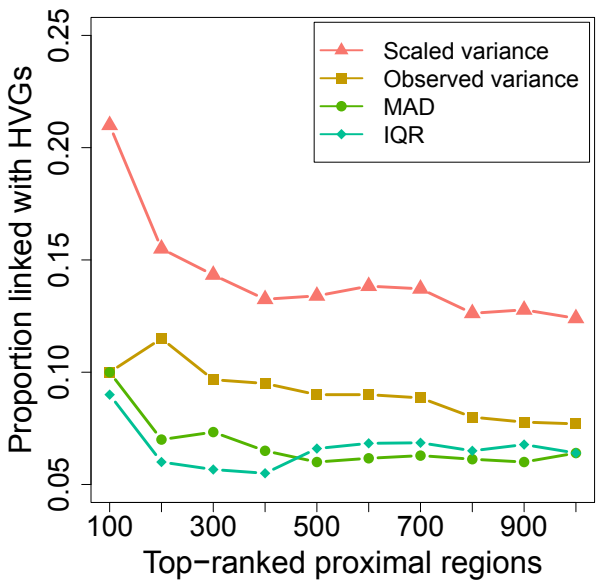

ATAC-seq

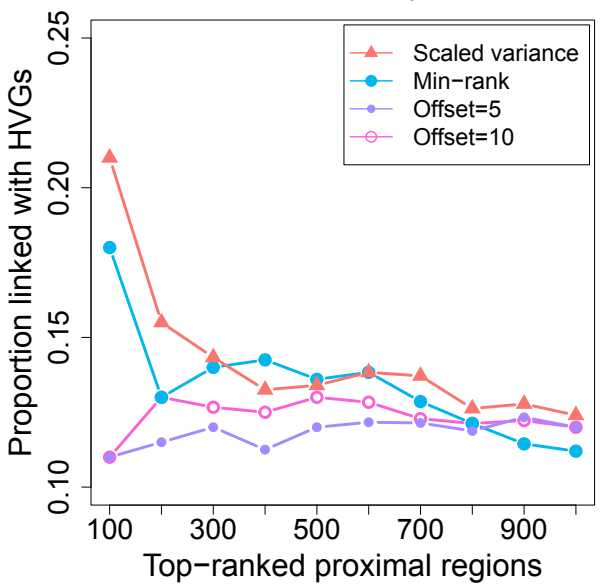

H3K27ac: proximal regions

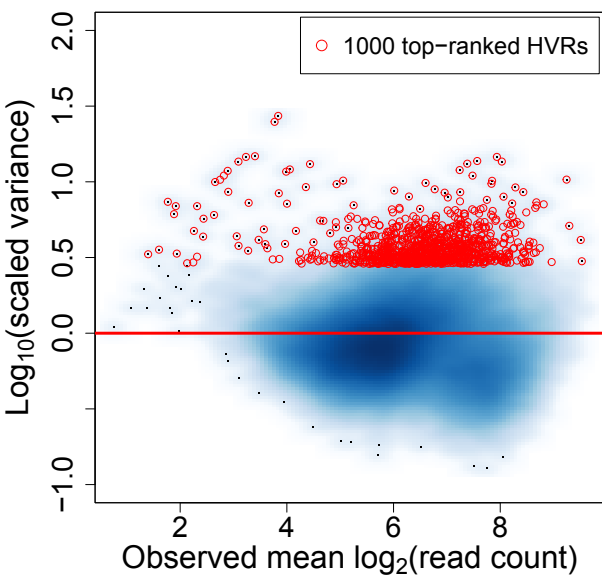

Pol II

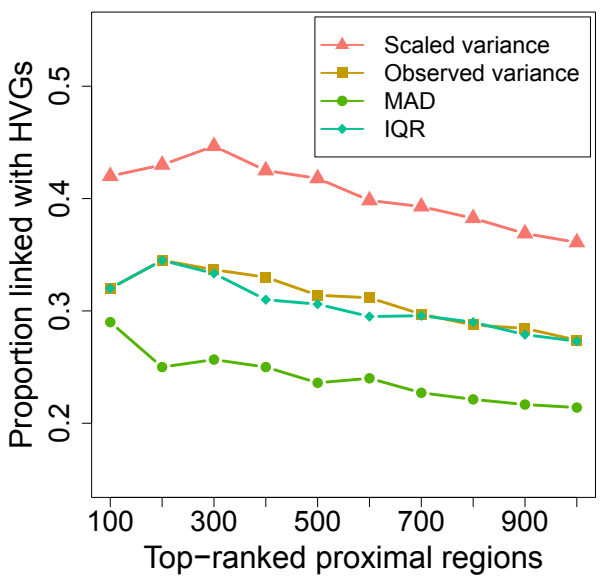

Pol II

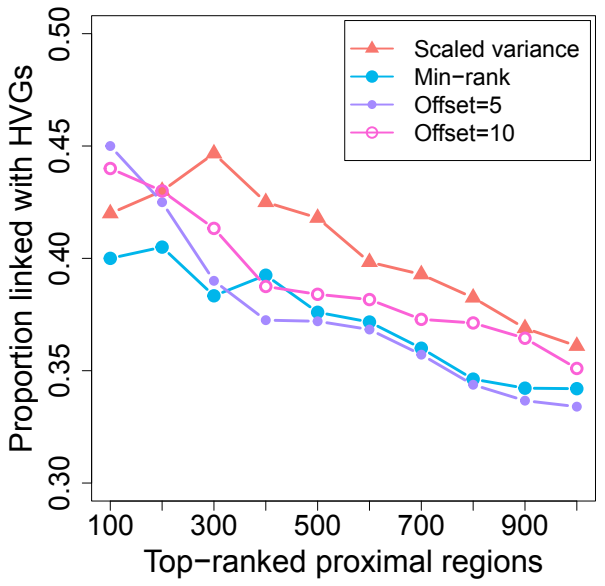


EiOu pron̂xiv preprint doi: https://doi.org/10.1101/2021.07.27.453915; this version posted July 27, 2021. The copyright holder for this preprint - IgUr was not certified by peer review) is the author/funder, who has granted bioRxiv a license to display the preprint in perpetuity. It is
made available under aCC-BY-NC-ND 4.0 International license.

A

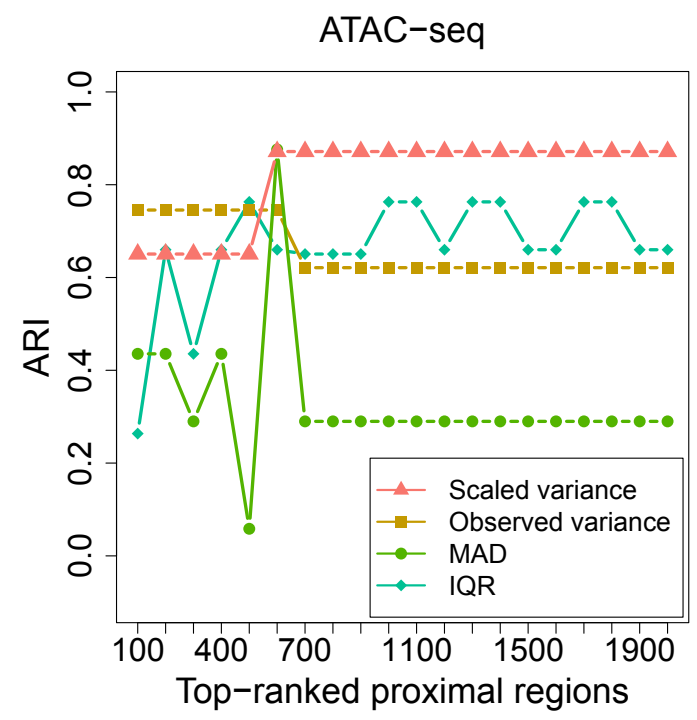

B

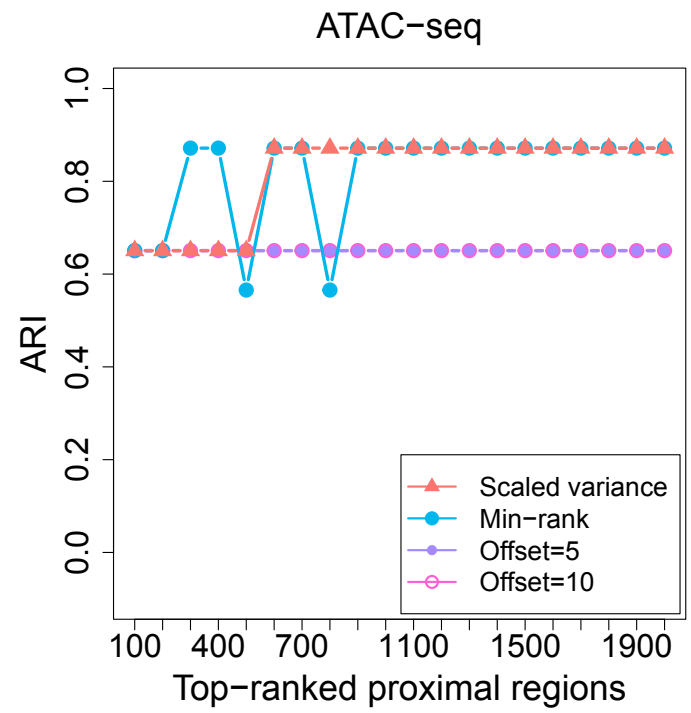

C

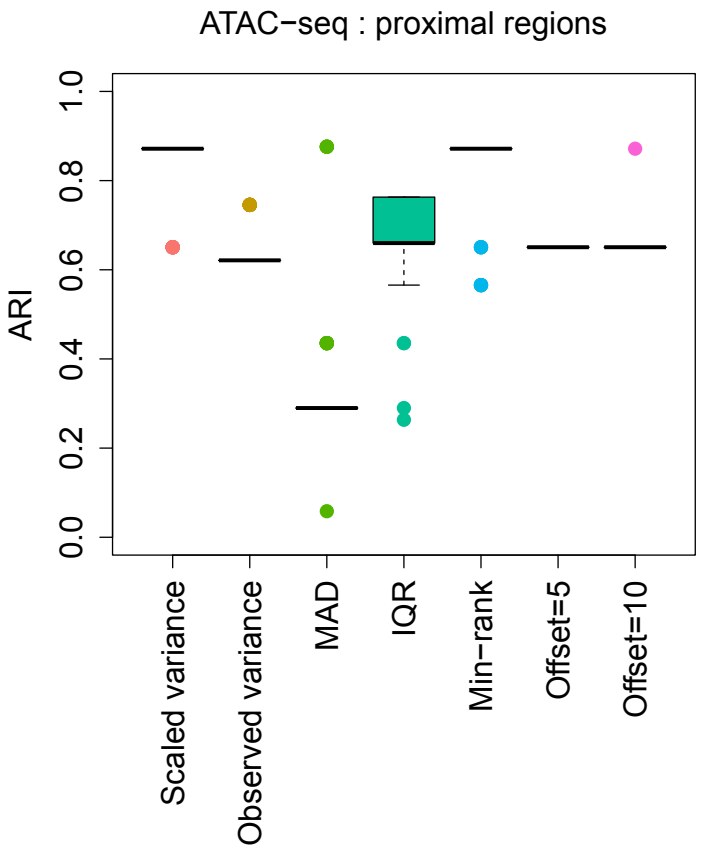

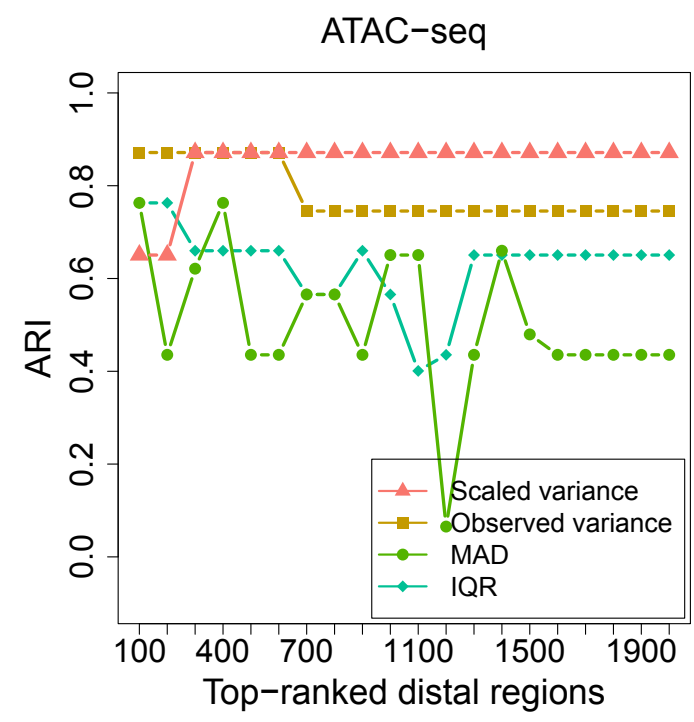
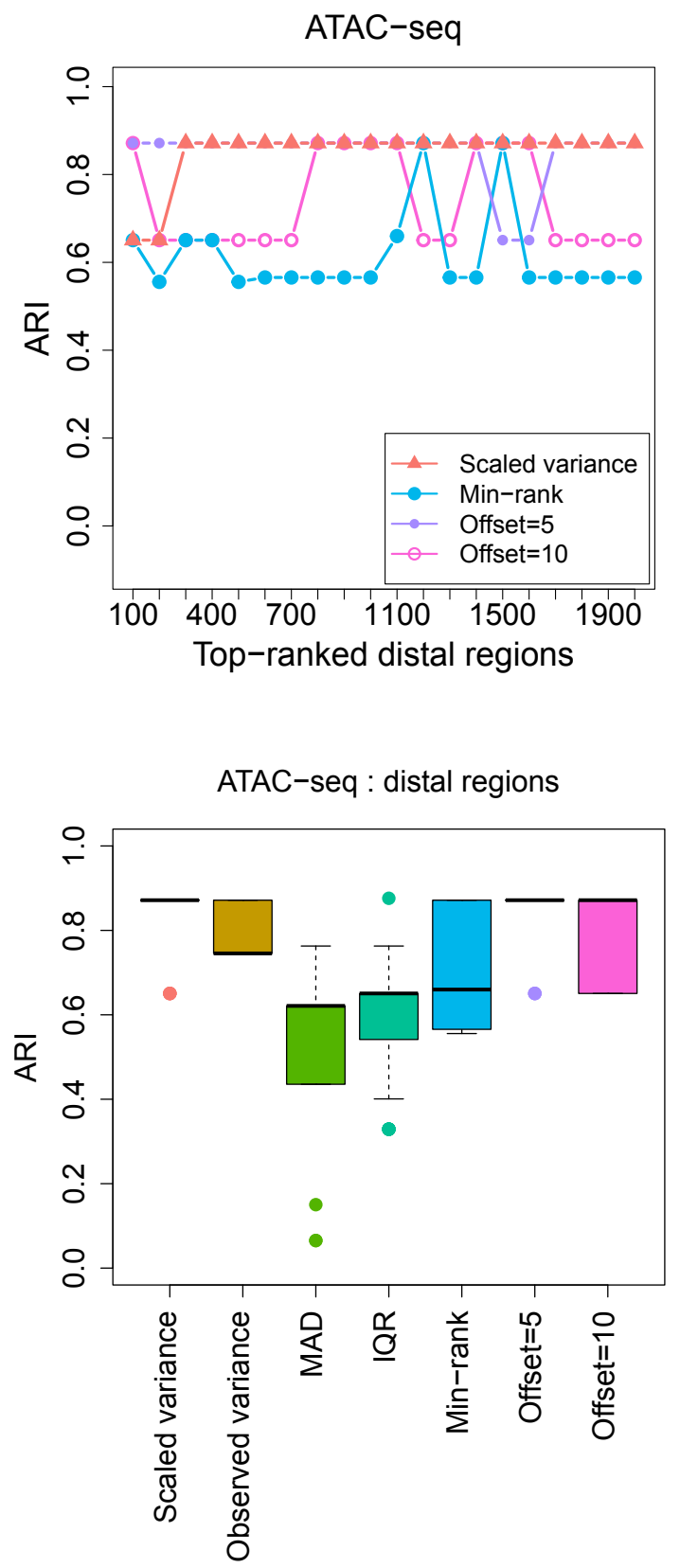
FiqureR3 preprint doi: https://doi.org/10.1101/2021.07.27.453915; this version posted July 27, 2021. The copyright holder for this preprint A made available under aCC-BY-NC-ND 4.0 International license.

B

H3K27ac: proximal regions
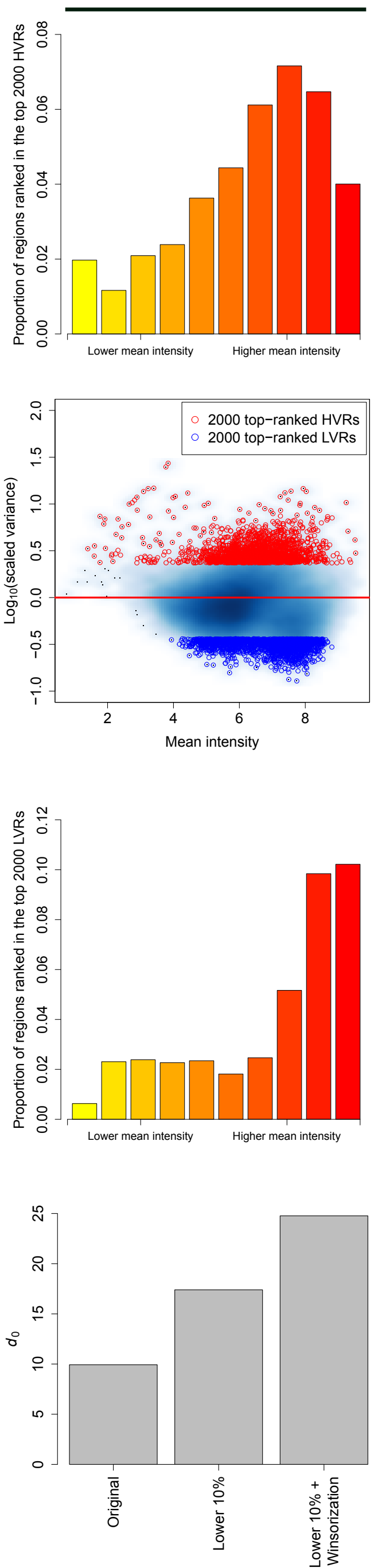

H3K27ac: distal regions
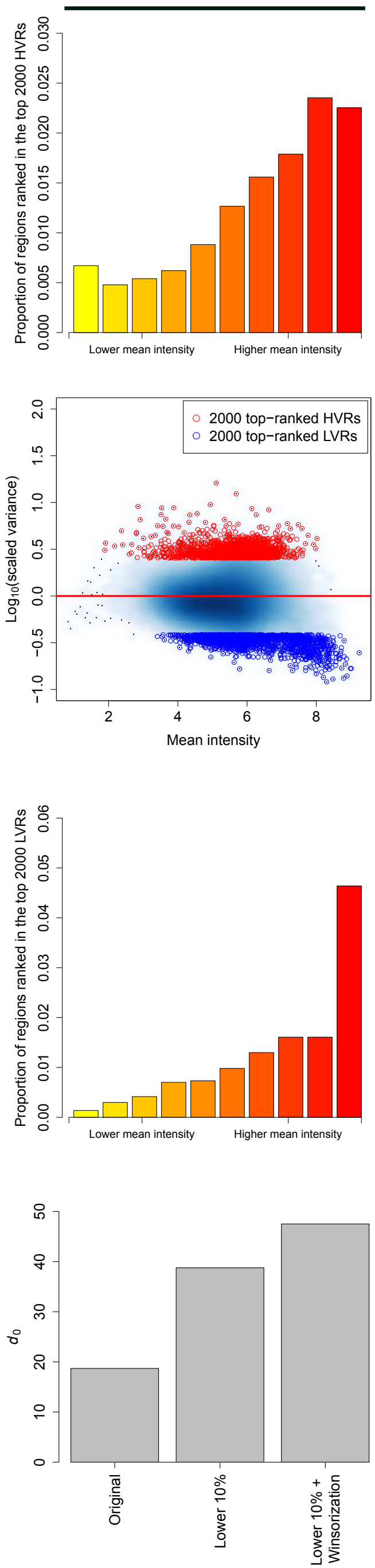


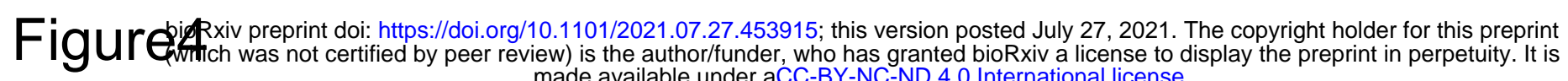

A

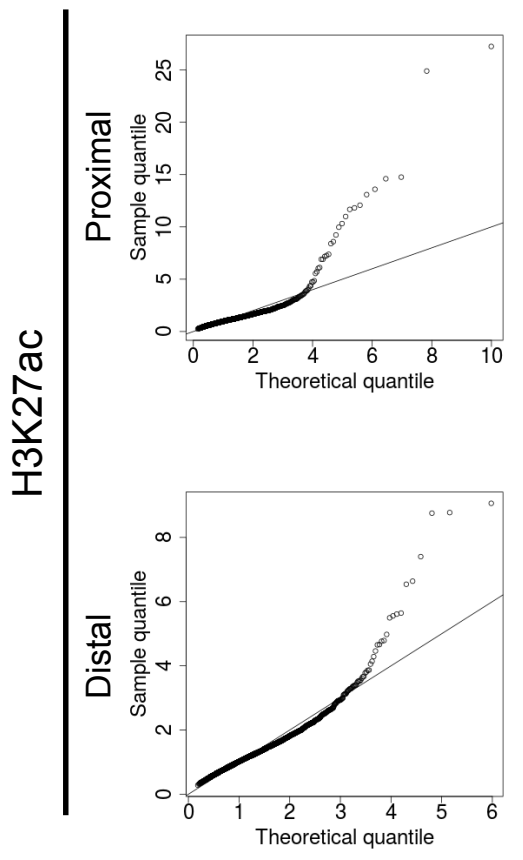

\begin{tabular}{l} 
\\
\hline \\
\hline
\end{tabular}
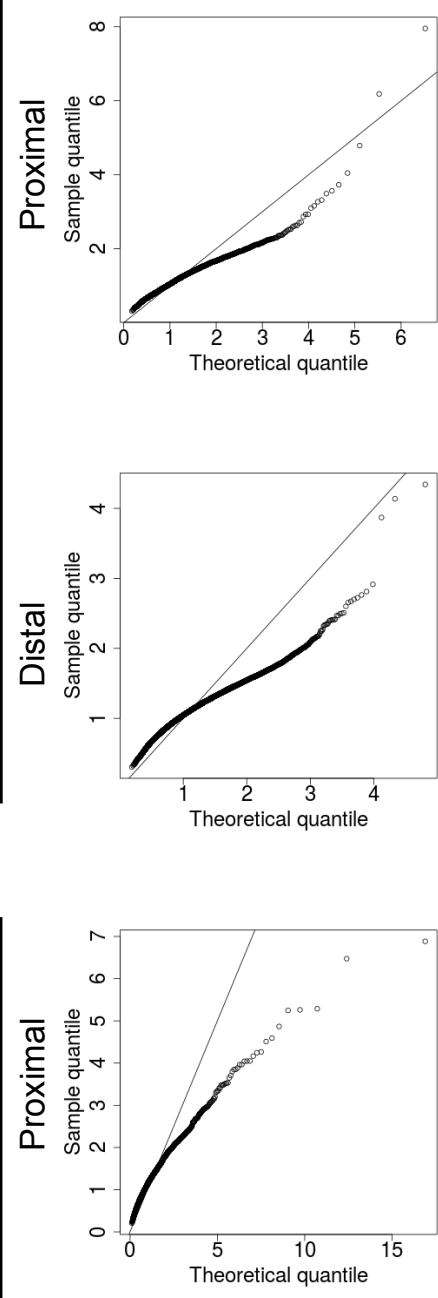

$\overline{\bar{O}}$

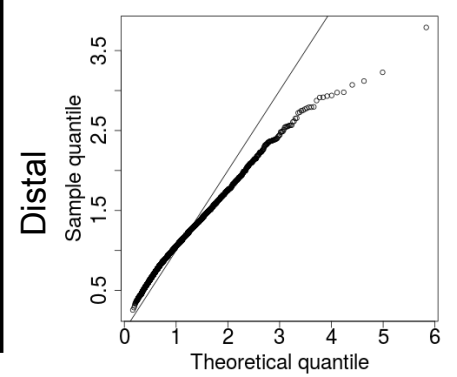

B
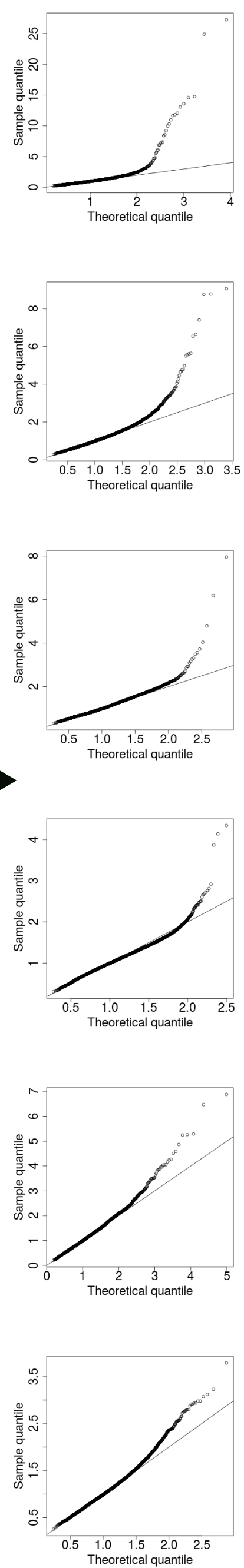

C
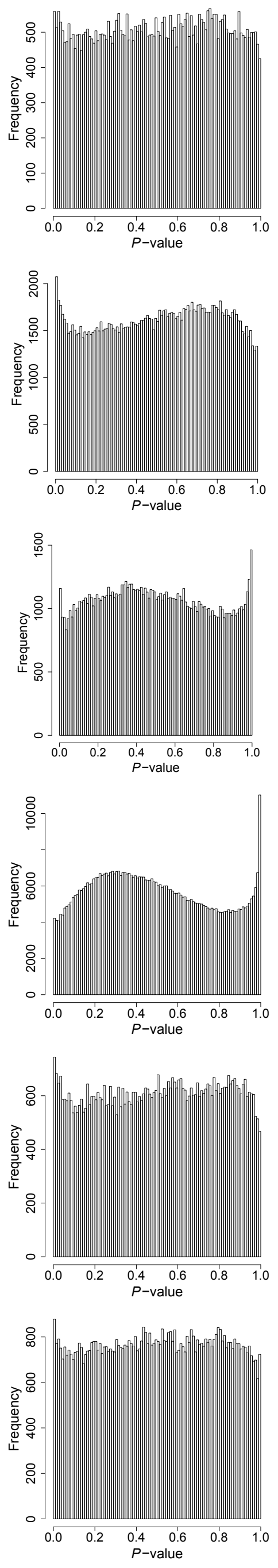

D
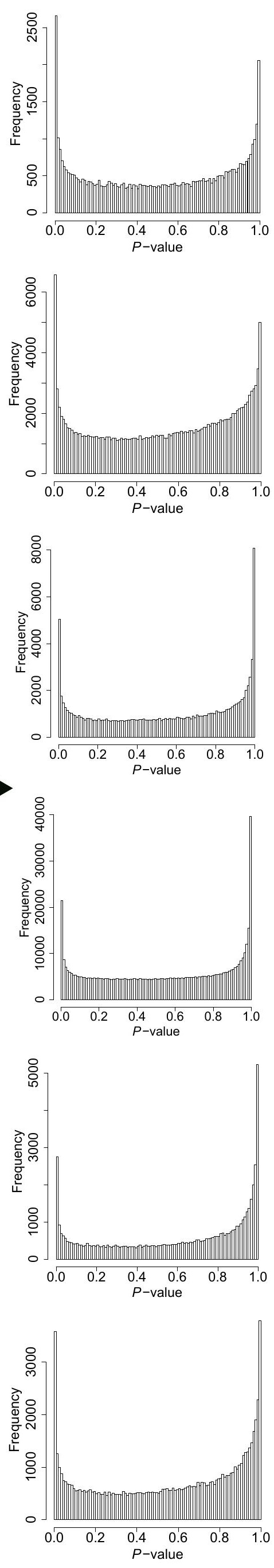
biqRxiv preprint doi: https://doi.org/10.1101/2021.07.27.453915; this version posted July 27, 2021. The copyright holder for this preprint Figuren was not certified by peer review) is the author/funder, who has granted bioRxiv a license to display the preprint in perpetuity. It is

A

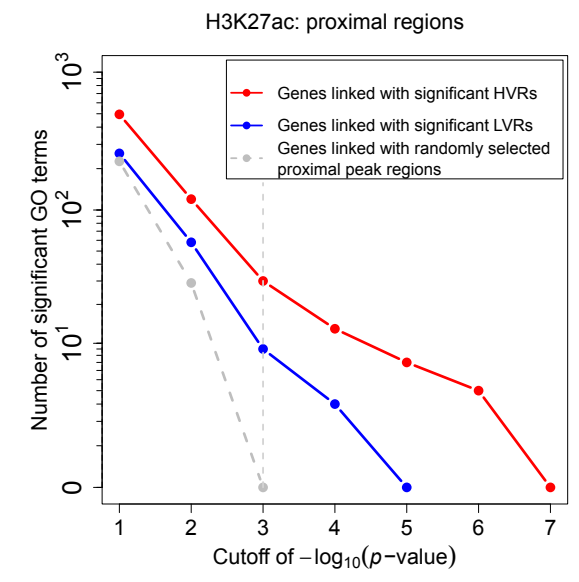

D

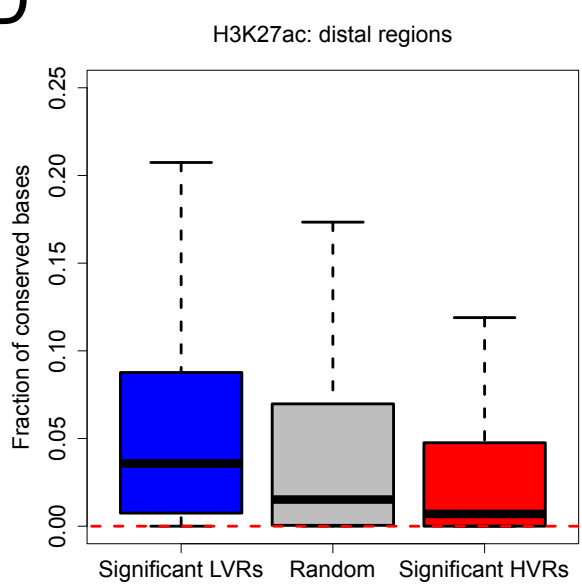

G

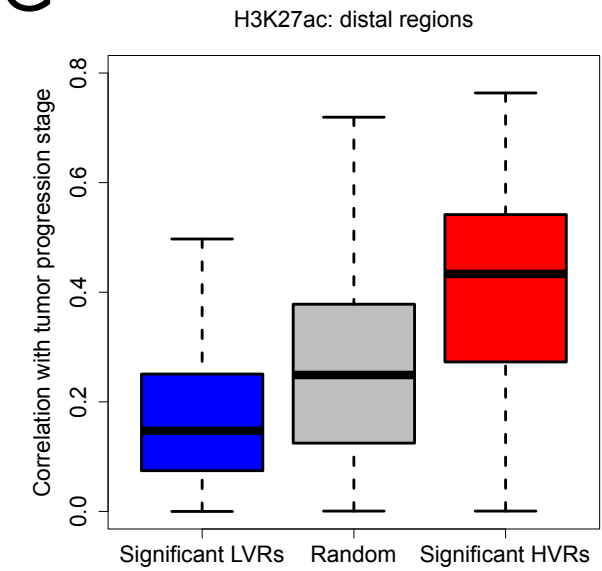

B

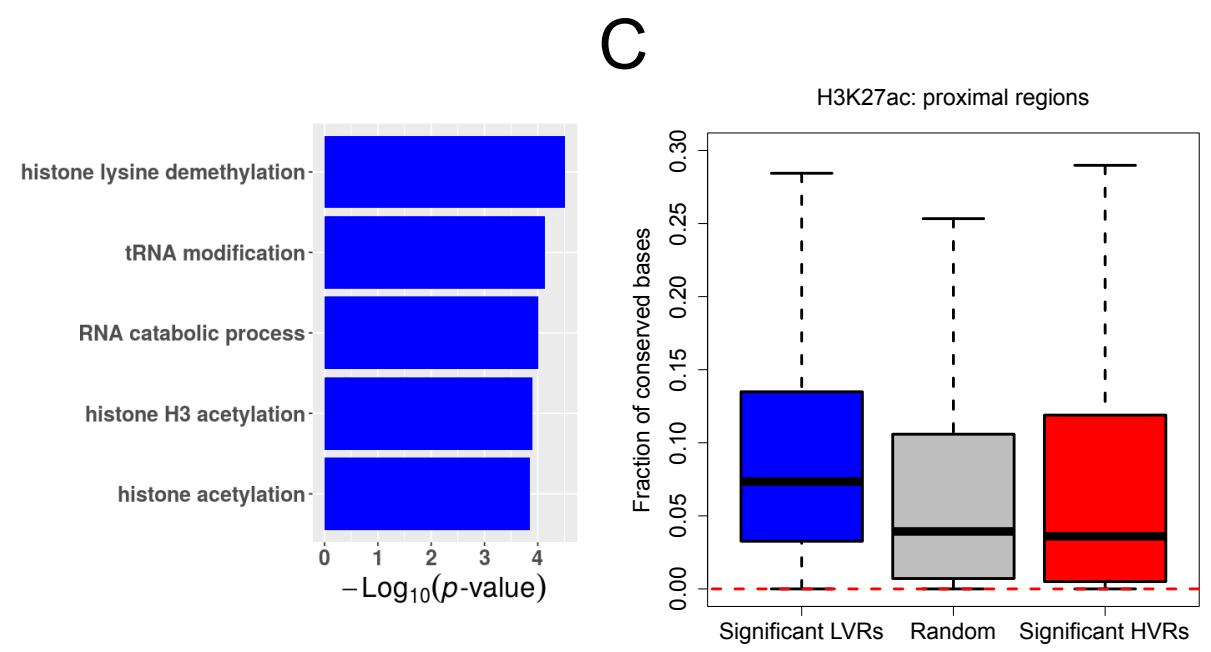

E

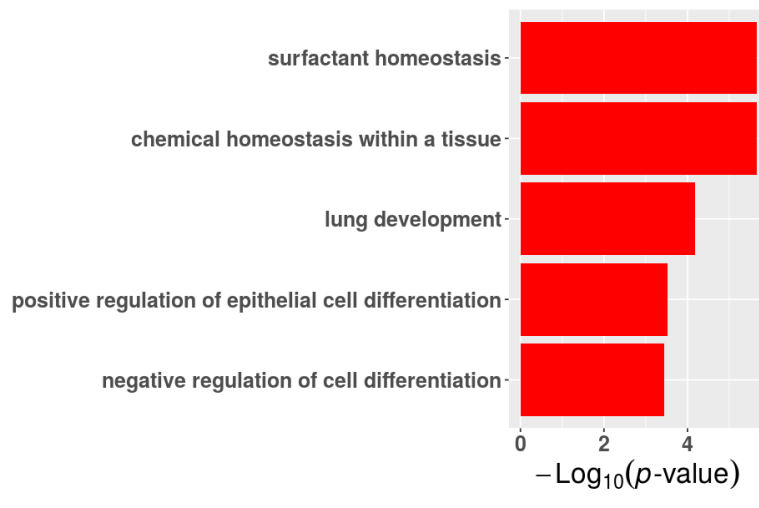

$\mathrm{H}$

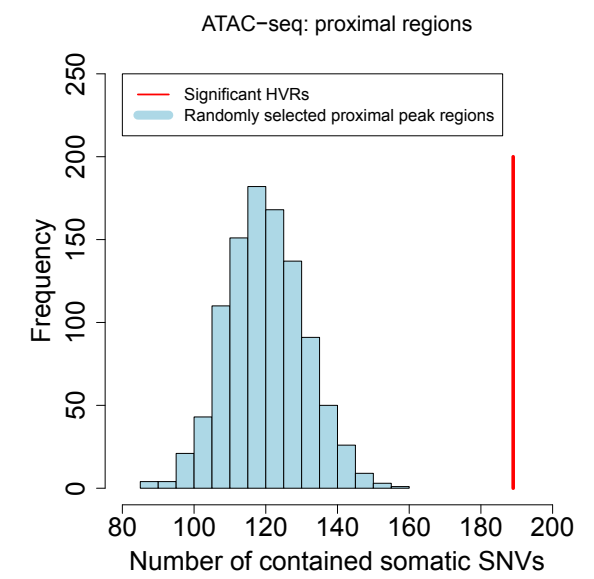

F

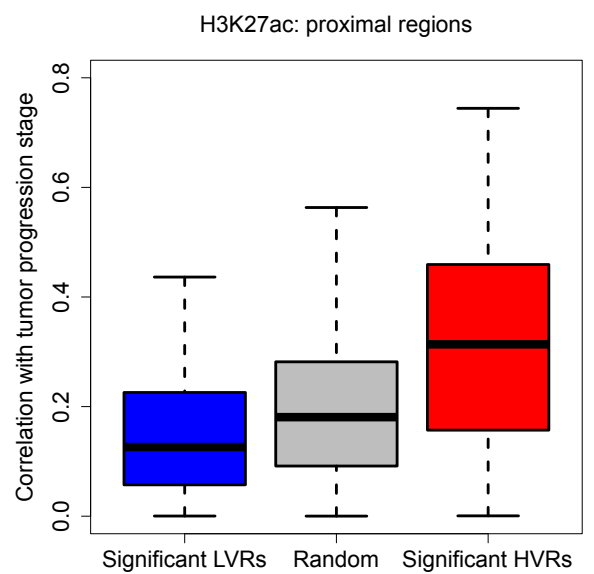

I

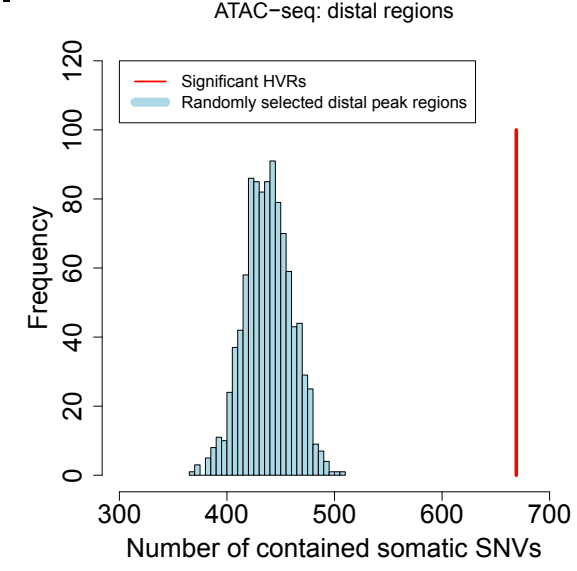


bioRxiv preprint doi: https://doi.org/10.1101/2021.07.27.453915; this version posted July 27, 2021. The copyright holder for this preprint (whie was not certified by peer review) is the author/funder, who has granted bioRxiv a license to display the preprint in perpetuity. It is Figurey was not certified by peer review) is the author/funder, who has granted bioRxiv a license to diste
made available under aCC-BY-NC-ND 4.0 International license.

A

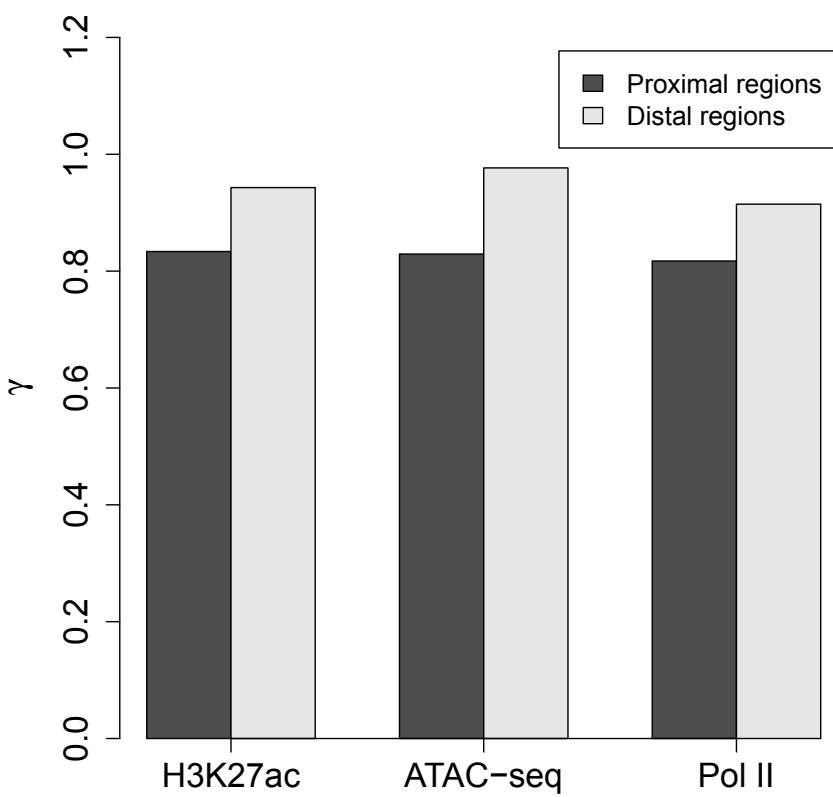

B

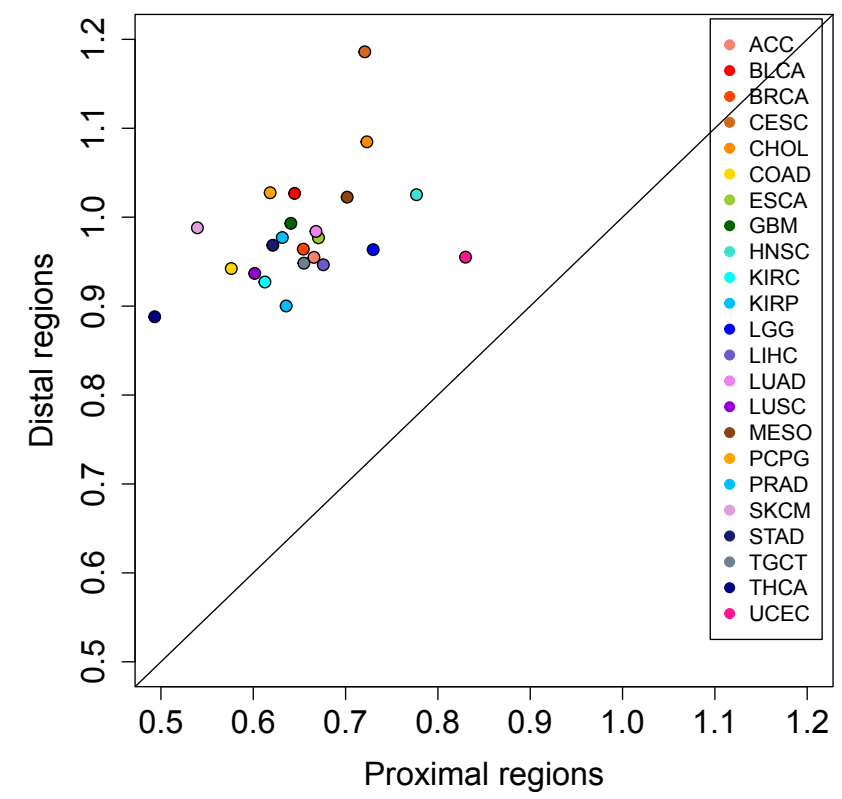

